\title{
GROUP SCHEMES OF PERIOD 2
}

\author{
VICTOR ABRASHKIN
}

\begin{abstract}
We give an explicit construction of the antiequivalence of the category of finite flat commutative group schemes of period 2 defined over a valuation ring of a 2-adic field with algebraically closed residue field. This result extends the earlier author's approach to group schemes of period $p>2$ from Proceedings LMS, 101, 2010, 207-259.
\end{abstract}

\section{INTRODUCTION}

0.1. Basic notation. Everywhere in the paper $k$ is algebraically closed field of characteristic $2, K_{00}$ is the fraction field of the ring of Witt vectors $W(k)$ and $\left[K_{0}: K_{00}\right]=e \in \mathbb{N}$. Let $O_{0}=O_{K_{0}}$ be the valuation ring of $K_{0}, \pi$ - a fixed uniformiser in $K_{0}, K=K_{0}(\pi)$, where $\pi^{2}=\pi_{0}$, and $O=O_{K}$. We set $S=k[[t]]$ where $t$ is a variable. Let $\sigma: S \longrightarrow S$ be such that $\sigma(s)=s^{2}, s \in S$. Denote by $\kappa_{S O}: S / t^{2 e} \longrightarrow O / 2 O$ the rings isomorphism such that $\left.\kappa_{S O}\right|_{k}=\mathrm{id}$ and $\kappa_{S O}: t \bmod t^{2 e} \mapsto \pi \bmod 2$.

For a natural number $u, \underline{i}$ denotes always a vector of length $u$ with coordinates from the set $\{0,1\}$ and $r(\underline{i})$ denotes the sum of these coordinates.

0.2. Categories of group schemes. Let $R$ be a local ring of characteristic 0 with residue field $k$. Denote by $\mathrm{Gr}_{R}$ the category of finite flat commutative group schemes $G$ over $R$ such that $2 \mathrm{id}_{G}=0$.

Recall that $G=\operatorname{Spec} A(G)$, where $A(G)$ is a flat $R$-algebra of finite rank $|G|$ and the structure of group scheme on $G$ is given via the $R$ algebra morphisms $e_{G}: A(G) \longrightarrow O$ (counit) and $\Delta_{G}: A(G) \longrightarrow$ $A(G) \otimes_{R} A(G)$ (coaddition) satisfying standard axioms.

Denote by $\mathrm{Gr}_{R}^{e t}$ and $\mathrm{Gr}_{R}^{\text {mult }}$ the full subcategories in $\mathrm{Gr}_{R}$ of etale and, resp., multiplicative group schemes. Then any $G \in \mathrm{Gr}_{R}$ has the maximal etale quotient $j^{e t}: G \longrightarrow G^{e t}$ and the maximal multiplicative subobject $i^{\text {mult }}: G^{\text {mult }} \longrightarrow G$.

Because $k$ is algebraically closed any etale object in $\mathrm{Gr}_{R}$ is a product of finitely many copies of the constant etale group scheme of order $2,(\mathbb{Z} / 2)_{O}=\operatorname{Spec} \operatorname{Map}(\mathbb{Z} / 2, R)$. Similarly, any multiplicative group scheme in $\mathrm{Gr}_{R}$ is a product of finitely many copies of the constant multiplicative group scheme of order $2, \mu_{2}=\operatorname{Spec} R[\mathbb{Z} / 2]$.

Date: Oct 31, 2011.

2010 Mathematics Subject Classification. 11S20, 14K10.

Key words and phrases. group schemes, local field. 
Introduce the category $\mathrm{Gr}_{R}^{*}$ as follows. Its objects are the objects from $\mathrm{Gr}_{R}$ and for any $G_{1}, G_{2} \in \mathrm{Gr}_{R}$,

$$
\operatorname{Hom}_{\mathrm{Gr}_{R}^{*}}\left(G_{1}, G_{2}\right)=\operatorname{Hom}_{\mathrm{Gr}_{R}}\left(G_{1}, G_{2}\right) / \mathcal{R}\left(G_{1}, G_{2}\right)
$$

where $\mathcal{R}\left(G_{1}, G_{2}\right)$ consists of the morphisms

$$
G_{1} \stackrel{j^{e t}}{\longrightarrow} G_{1}^{e t} \stackrel{f}{\longrightarrow} G_{2}^{\text {mult }} \stackrel{i^{\text {mult }}}{\longrightarrow} G_{2}
$$

whith arbitrary $f \in \operatorname{Hom}_{\operatorname{Gr}_{R}}\left(G_{1}^{\text {et }}, G_{2}^{\text {mult }}\right)$. Note that $\operatorname{Hom}_{\mathrm{Gr}_{R}}\left((\mathbb{Z} / 2)_{R}, \mu_{2, R}\right)$ has only one non-trivial element given by the embedding of $R$-algebras

$$
R[\mathbb{Z} / 2]=R \overline{0}+R \overline{1} \longrightarrow \operatorname{Map}(\mathbb{Z} / 2, R)=R \oplus R
$$

such that $\overline{0} \mapsto(1,1)$ and $\overline{1} \mapsto(1,-1)$.

0.3. Categories of filtered modules. Let $\mathcal{M F}_{S}$ be the category of the triples $\left(M^{0}, M^{1}, \varphi_{1}\right)$ such that $M^{1} \subset M^{0}$ are $S$-modules and $\varphi_{1}$ : $M^{1} \longrightarrow M^{0}$ is a $\sigma$-linear morphism of $S$-modules. The morphisms in $\mathcal{M F}_{S}$ are compatible morphisms of $S$-modules commuting with $\varphi_{1}$.

Denote by $\mathrm{MF}_{S}^{e}$ the full subcategory in $\mathcal{M F}_{S}$ consisting of the triples $\left(M^{0}, M^{1}, \varphi_{1}\right)$ such that

- $M^{0}$ is a free $S$-module of finite rank;

- $M^{1} \supset t^{e} M^{0}$

- $\varphi_{1}\left(M^{1}\right) S=M^{0}$.

The full subcategory of etale filtered modules $\mathrm{MF}_{S}^{e, e t}$ in $\mathrm{MF}_{S}^{e}$ consists of $\left(M^{0}, M^{1}, \varphi_{1}\right)$ such that $M^{1}=t^{e} M^{0}$. One can see easily that any $\mathcal{M}=\left(M^{0}, M^{1}, \varphi_{1}\right) \in \mathrm{MF}_{S}^{e}$ has a unique maximal etale subobject $i^{e t}: \mathcal{M}^{e t}=\left(M^{0, e t}, t^{e} M^{0, e t}, \varphi_{1}\right) \longrightarrow \mathcal{M}$. Suppose $\varphi_{0}: M^{0} \longrightarrow M^{0}$ is such that $\varphi_{0}(m):=\varphi_{1}\left(t^{e} m\right)$ for any $m \in M^{0}$. Then $\bar{M}^{0, e t}:=M^{0, e t} \otimes_{S} k$ is the maximal $k$-submodule of $\bar{M}^{0}:=M^{0} \otimes_{S} k$ such that $\varphi_{0}$ induces an invertible $\sigma$-linear automorphism on $\bar{M}^{0, e t}$. Notice that $i^{\text {et }}$ can be included into the following short exact sequence

$$
0 \longrightarrow \mathcal{M}^{e t} \stackrel{i^{e t}}{\longrightarrow} \mathcal{M} \longrightarrow \mathcal{M}^{l o c} \longrightarrow 0
$$

where $\mathcal{M}^{l o c}=\left(M^{0, l o c}, M^{1, l o c}, \varphi_{1}\right) \in \mathrm{MF}_{S}^{e}$ and $\bar{M}^{0, l o c}:=M^{0, l o c} \otimes_{S} k$ can be naturally indentified with the maximal $k$-submodule in $\bar{M}^{0}$ such that $\varphi_{0}$ induces its nilpotent endomorphism. Any etale filtered module is a direct sum of finitely many copies of $\mathcal{S}^{e t}:=\left(S m, S t^{e} m, \varphi_{1}\right) \in \mathrm{MF}_{S}^{e, e t}$, where $\varphi_{1}\left(t^{e} m\right)=m$.

The full subcategory of multiplicative filtered modules $\mathrm{MF}_{S}^{e, m u l t}$ in $\mathrm{MF}_{S}^{e}$ consists of $\left(M^{0}, M^{1}, \varphi_{1}\right)$ such that $M^{1}=M^{0}$. Any $\mathcal{M} \in \mathrm{MF}_{S}^{e}$ has a unique maximal multiplicative quotient $j^{\text {mult }}: \mathcal{M} \longrightarrow \mathcal{M}^{\text {mult }}$. Introduce the morphism $\psi_{0}: \bar{M}^{0} \longrightarrow \bar{M}^{0}$ as follows: if $m \in M^{1}$ and $\varphi_{1}(m)=n \in M^{0}$ then $\psi_{0}(n \otimes 1)=m \otimes 1$. One can verify that $\psi_{0}$ is a well-defined $\sigma^{-1}$-linear morphism of $k$-modules and $\bar{M}^{0, \text { mult }}:=$ $M^{0, m u l t} \otimes_{S} k$ can be identified with the maximal $k$-submodule in $\bar{M}^{0}$ 
such that $\left.\psi_{0}\right|_{\bar{M}^{0, m u l t}}$ is invertible. Note that $j^{\text {mult }}$ can be included into the following short exact sequence in the category $\mathrm{MF}_{S}^{e}$,

$$
0 \longrightarrow \mathcal{M}^{u} \longrightarrow \mathcal{M} \stackrel{j^{\text {mult }}}{\longrightarrow} \mathcal{M}^{\text {mult }} \longrightarrow 0
$$

where $\mathcal{M}^{u}=\left(M^{0, u}, M^{1, u}, \varphi_{1}\right) \in \mathrm{MF}_{S}^{e}$ and $\bar{M}^{0, u}:=M^{0, u} \otimes_{S} k$ is the maximal $k$-submodule such that $\left.\psi_{0}\right|_{\bar{M}^{0, u}}$ is nilpotent. Any multiplicative filtered module is a direct sum of finitely many copies of $\mathcal{S}^{\text {mult }}:=\left(S n, S n, \varphi_{1}\right) \in \mathrm{MF}_{S}^{e, \text { mult }}$, where $\varphi_{1}(n)=n$.

Introduce the category $\mathrm{MF}_{S}^{e *}$ as follows. Its objects are the objects of $\mathrm{MF}_{S}^{e}$ and for any $\mathcal{M}_{1}, \mathcal{M}_{2} \in \mathrm{MF}_{S}^{e}$,

$$
\operatorname{Hom}_{\mathrm{MF}_{S}^{e *}}\left(\mathcal{M}_{1}, \mathcal{M}_{2}\right)=\operatorname{Hom}_{\mathrm{MF}_{S}^{e}}\left(\mathcal{M}_{1}, \mathcal{M}_{2}\right) / R\left(\mathcal{M}_{1}, \mathcal{M}_{2}\right)
$$

where $R\left(\mathcal{M}_{1}, \mathcal{M}_{2}\right)$ consists of the morphisms of $\mathrm{MF}_{S}^{e}$ of the form

$$
\mathcal{M}_{1} \stackrel{j^{\text {mult }}}{\longrightarrow} \mathcal{M}_{1}^{\text {mult }} \stackrel{f}{\longrightarrow} \mathcal{M}_{2}^{\text {et }} \stackrel{i^{e t}}{\longrightarrow} \mathcal{M}_{2}
$$

with arbitrary $f \in \operatorname{Hom}_{\mathrm{MF}_{S}^{e}}\left(\mathcal{M}_{1}^{\text {mult }}, \mathcal{M}_{2}^{\text {et }}\right)$.

Note that $\operatorname{Hom}_{\mathrm{MF}_{S}^{e}}\left(\mathcal{S}^{\text {mult }}, \mathcal{S}^{\text {et }}\right)$ has only one non-trivial morphism and it is given by the correspondence $n \mapsto t^{2 e} m$.

0.4. Main result. In this paper we prove the following theorem.

Theorem 0.1. There is an antiequivalence of categories

$$
\mathcal{F}_{O_{0}}^{O *}: \mathrm{Gr}_{O_{0}}^{*} \longrightarrow \mathrm{MF}_{S}^{e *}
$$

For $p>2$, there is an antiequivalence of categories $\mathcal{F}_{O_{0}}^{O}: \mathrm{Gr}_{O_{0}} \longrightarrow$ $\mathrm{MF}_{S}^{e}$. This was proved by C.Breuil [5] and M.Kisin [7, 8] in a more general context of all $p$-group schemes. The proofs are obtained from the study of $p$-divisible groups and essentially use the crystalline Dieudonne theory which is built on a geometrical approach due to the Raynaud theorem about the existence of embedding of any $p$-divisible group into an abelian scheme. This approach has been generalized recently by W.Kim [6], E.Lau [9] and T.Liu [10] to the case $p=2$. (Lau's result uses Zink's theory of displays and windows.)

On the other hand, an explicit and direct construction of the antiequivalence $\mathcal{F}_{O_{0}}^{O}$ in the case $p>2$ was given by the author [4]. The above theorem extends that construction to the case $p=2$. We should notice that this extension is very far from to be straightforward for the following reasons.

First, when relating group schemes over $O_{0} \subset O$ and filtered $S$ modules we use the identification of rings $S / t^{2 e}$ and $O / 2$. But when working modulo 2 we can't control quite efficiently all morphisms in the category $\operatorname{Gr}_{O}$, e.g. both the elements of $\operatorname{Hom}_{\mathrm{Gr}_{O}}\left((\mathbb{Z} / 2)_{O}, \mu_{2, O}\right)$ coincide modulo 2O. This explains why we are forced to use the quotient categories $\mathrm{Gr}_{O}^{*}, \mathrm{Gr}_{O_{0}}^{*}$ and $\mathrm{MF}_{S}^{e *}$. On the other hand, the above example represents essentially the only aspect we are losing in our approach and 
Theorem 0.1 gives essentially complete information about the objects and morphisms of the category $\mathrm{Gr}_{O_{0}}$.

Second, when working with odd prime numbers $p>2$ the ideal $p O$ is provided with nilpotent $D P$-structure and is considerably smaller than the maximal $D P$-ideal in $O$ if $e>1$. If $p=2$ we have no such "safety margin" because $2 O$ is already the maximal $D P$-ideal in $O$. The adjustment of methods of [4] to the case $p=2$ required a profound revision of all constructions used in there, especially the proof of the surjectivity of the functor $\mathcal{G}_{O_{0}}^{O}$. In particular, so-called Main Lemma was restated in a more precise form and provided with an essential elaboration. As a result, all main features of our approach from [4] were preserved in the case $p=2$.

Finally, notice that all applications developed in [4]: a criterion for the Galois module to come from the Galois module of geometric points of $G \in \mathrm{Gr}_{O_{0}}$, the relation between group schemes from $\mathrm{Gr}_{O_{0}}$ and Faltings's strict modules in characteristic 2 and an explicit description of the duality in $\mathrm{Gr}_{O_{0}}$ can be done along the lines of the approach from [4] in the case $p=2$ as well.

0.5. Brief description of used methods. Let $\mathrm{Aug}_{O}$ be the category of augmented $O$-algebras. Introduce the equivalence relation $R$ such that for $f_{1}, f_{2} \in \operatorname{Hom}_{\text {Aug }_{O}}\left(\mathcal{B}_{1}, \mathcal{B}_{2}\right), f_{1} \underset{R}{\sim} f_{2}$ iff $f_{1}$ and $f_{2}$ coincide modulo some DP-ideal in $\mathcal{B}_{2}$ (cf. Subsection 1.3 for the definition of this ideal). Denote by $\operatorname{Aug}_{O}^{*}$ the category whose objects are the objects of $\mathrm{Aug}_{O}$ but the morphisms are the $R$-equivalence classes of morphisms in $\mathrm{Aug}_{O}$. In our approach the category $\operatorname{Aug}_{O}\left(\operatorname{resp}, \operatorname{Aug}_{O}^{*}\right.$ ) relates the categories $\mathrm{MF}_{S}^{e}$ and $\mathrm{Gr}_{O}$ (resp., $\mathrm{MF}_{S}^{e *}$ and $\mathrm{Gr}_{O}^{*}$ ).

As first step we associate with $\mathcal{M}=\left(M^{0}, M^{1}, \varphi_{1}\right) \in \mathrm{MF}_{S}^{e}$ a set of augmented $O$-algebras $\operatorname{Aug}_{O}(\mathcal{M})$ each of whose members is constructed after choosing an appropriate special basis for $M^{0}$ and a couple of other choices. One also defines for any $\mathcal{A} \in \mathrm{Aug}_{O}$, a canonical object $\iota(\mathcal{A}) \in \mathcal{M F}_{S}$ and if $\mathcal{A} \in \operatorname{Aug}_{O}(\mathcal{M})$ then we have a natural map $\iota_{\mathcal{M}}$ : $\mathcal{M} \longrightarrow \iota(\mathcal{A})$ in $\mathcal{M F}_{S}$. Then one observes that the correspondences $\mathcal{A} \mapsto \operatorname{Aug}_{O}(\mathcal{M})$ and $\mathcal{B} \mapsto \iota(\mathcal{B})$ are left-adjoint. This results in the following property: if $\mathcal{N} \in \mathrm{MF}_{S}^{e}$ and $\mathcal{B} \in \operatorname{Aug}_{O}(\mathcal{N})$ then we have natural identifications

$$
\operatorname{Hom}_{M_{S}^{e *}}^{*}(\mathcal{M}, \mathcal{N})=\operatorname{Hom}_{\mathcal{M} \mathcal{F}_{S}}\left(\iota_{\mathcal{M}}(\mathcal{M}), \iota_{\mathcal{N}}(\mathcal{N})\right) \subset \operatorname{Hom}_{\text {Aug }_{O}^{*}}(\mathcal{A}, \mathcal{B}) .
$$

This allows us to show that $\mathcal{A}$, as an object of $\operatorname{Aug}_{O}^{*}$, is functorial in $\mathcal{M}$ (viewed as an object of $\mathrm{MF}^{e *}$ ), and that the assignment $\mathcal{M} \mapsto \mathcal{A}$ is functorial. It also allows us to define a family $\mathcal{L}_{\mathcal{A}}$ of Hopf algebra structures on $\mathcal{A}$ whose spectrums are group schemes over $O$ that are isomorphic in $\mathrm{Gr}_{O}^{*}$. (Use the diagonal embedding of $\mathcal{M}$ into $\mathcal{N}=$ $\mathcal{M} \oplus \mathcal{M}$.) In this way we obtain a faithful functor $\mathcal{G}_{O}: \mathrm{MF}_{S}^{e *} \longrightarrow \mathrm{Gr}_{O}^{*}$. 
This functor is actually full. To see this, we describe $\iota_{\mathcal{M}}(\mathcal{M})$ in terms of the Hopf algebra structure on $\mathcal{A}$, and so we find that it is an object intrinsically attached to $\mathcal{G}_{O}(\mathcal{M})$. So we obtain the following commutative diagram:

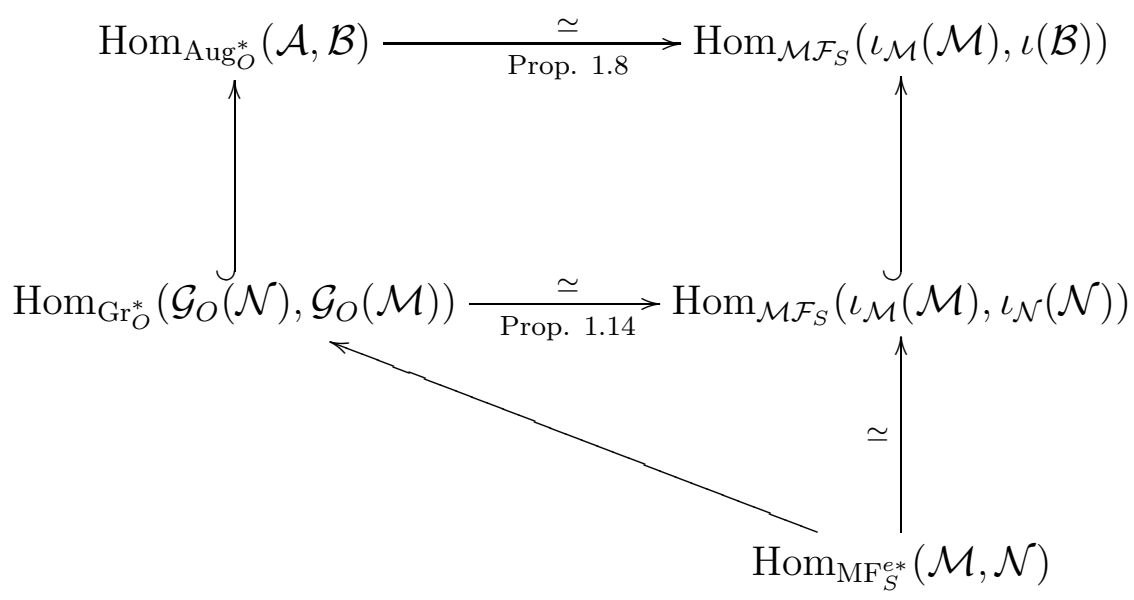

These ideas have been applied earlier by the author in [2, 3] to describe the category $\mathrm{Gr}_{O}^{*}$, where $O$ is the valuation ring of a field extension of $\mathbb{Q}_{p}$ with small ramification.

Next, one shows that $\mathcal{G}_{O}$ factors through a functor $\mathcal{G}_{O_{0}}^{O}: \mathrm{MF}_{S}^{e *} \longrightarrow$ $\mathrm{Gr}_{O_{0}}^{*}$. This amounts to showing that every group scheme $\mathcal{G}_{O}(\mathcal{M})$ descends to one over $O_{0}$. To accomplish this one uses tame descent and induction on rank of $\mathcal{M}$. This goes more or less along the lines of [4].

All that remains to do is to show that $\mathcal{G}_{O_{0}}^{O}$ is essentially surjective. One again uses tame descent and induction, this time on $\left|G_{0}\right|=p^{s}$. This is most difficult part of the paper, where we need essential elaboration of Main Lemma from [4] and where a special role of prime number $p=2$ can be explained in the following way. When applying induction on $s$ we present $G=G_{0} \otimes_{O_{0}} O$ as an extension of a group scheme $H$ of order $p^{s-1}$ via a group scheme of order $p$. Then on the level of algebras $A(G)$ and $A(H)$ of the group schemes $G$ and $H$, the Kummer theory provides us with a class of generators $\theta$ such that $A(G)=A(H)[\theta]$. Main Lemma allows us to make a very special choice of $\theta$ and then via the Lubin-Tate logarithm $l_{L T}(X)=X+X^{2} / p+X^{p^{2}} / p^{2}+\ldots$, we can relate $G$ with a group scheme of the form $\mathcal{G}_{O}(\mathcal{M}), \mathcal{M} \in \mathrm{MF}_{S}^{e}$. This special choice becomes much more delicate in the case $p=2$ because when $p>2$ it was enough to use only the first two terms of the above expansion of $l_{L T}$ but in the case $p=2$ we need to take into account one term more. 


\section{Construction of the functor $\mathcal{G}_{O}: \mathrm{MF}_{S}^{e} \longrightarrow \mathrm{Gr}_{O}$}

We use all notation and assumptions from Introduction, in particular, the definition of the category $\mathrm{MF}_{S}^{e}$ and the corresponding properties of its objects. Remind also that we fixed an identification $\kappa_{S O}$ : $S / t^{2 e} S \simeq O / 2 O$ such that $\left.\kappa_{S O}\right|_{k}=$ id and $\kappa_{S O}\left(t \bmod t^{2 e}\right)=\pi \bmod 2$.

1.1. $\varphi_{1}$-lifts. Suppose $\mathcal{M}=\left(M^{0}, M^{1}, \varphi_{1}\right) \in \mathrm{MF}_{S}^{e}, \mathcal{N}=\left(N^{0}, N^{1}, \varphi_{1}\right) \in$ $\mathcal{M F}_{S}$ and $\theta \in \operatorname{Hom}_{\mathcal{M} \mathcal{F}_{S}}(\mathcal{M}, \mathcal{N})$.

Definition. a) $\theta$ is a $\varphi_{1}$-lift if $\theta\left(M^{0}\right)=N^{0}, \theta\left(M^{1}\right)=N^{1}$ and $\operatorname{Ker} \theta=$ $\left.\operatorname{Ker} \theta\right|_{M^{1}}:=T$; b) $\varphi_{1}$-lift $\theta$ is nilpotent if $\left.\varphi_{1}\right|_{T}$ is topologically nilpotent, i.e. $\bigcap_{n} \varphi_{1}^{n}(T)=0$; c) $\varphi_{1}$-lift $\theta$ is special if there is a submodule $T^{\prime}$ of $T$ such that $T=T^{\prime}+\left(T \cap t^{2 e} M^{0, e t}\right)$ and $\varphi_{1}$ induces a topologically nilpotent endomorphism of $T^{\prime}$.

Proposition 1.1. Suppose for $i=1,2, \theta_{i} \in \operatorname{Hom}_{\mathcal{M F}_{S}}\left(\mathcal{M}_{i}, \mathcal{N}_{i}\right)$ are $\varphi_{1}$-lifts and $h \in \operatorname{Hom}_{\mathcal{M F}_{S}}\left(\mathcal{N}_{1}, \mathcal{N}_{2}\right)$. Then the set $\mathcal{L}(h)$ of all $f \in$ $\operatorname{Hom}_{\mathrm{MF}_{S}^{e}}\left(\mathcal{M}_{1}, \mathcal{M}_{2}\right)$ such that $\theta_{2} \circ f=h \circ \theta_{1}$ is not empty. If in addition:

a) $\theta_{2}$ is nilpotent then $\mathcal{L}(h)$ consists only of one element;

b) $\theta_{2}$ is special then all elements of $\mathcal{L}(h)$ belong to $R\left(\mathcal{M}_{1}, \mathcal{M}_{2}\right)$, i.e. coincide in $\mathrm{MF}_{S}^{e *}$.

Proof. We start with the following Lemma.

Lemma 1.2. Suppose $L$ is a finitely generated $S$-module and $\varphi$ is a $\sigma$-linear operator on $L$. Then the operator $\mathrm{id}_{L}-\varphi$ is epimorphic. If, in addition, $\varphi$ is topologically nilpotent then $\operatorname{id}_{L}-\mathcal{A}$ is bijective.

Proof of Lemma. Part b) is obvious. In order to prove a) notice first that we can replace $L$ by $L / t L$ and, therefore, assume that $L$ is a finite dimensional vector space over $k$. Then there is a decomposition $L=$ $L_{1} \oplus L_{2}$, where $\varphi$ is invertible on $L_{1}$ and nilpotent on $L_{2}$. It remains to note that $L_{1}=L_{0} \otimes_{\mathbb{F}_{p}} k$, where $L_{0}$ is a finite dimensional $\mathbb{F}_{p}$-vector space such that $\left.\varphi\right|_{L_{0}}=\mathrm{id}$. The existence of $L_{0}$ is a standard fact of $\sigma$-linear algebra: if $s=\operatorname{dim}_{k} L_{1}$ and $A \in M_{s}(k)$ is a matrix of $\left.\varphi\right|_{L_{1}}$ in some $k$ basis of $L_{1}$ then $L_{0}=\left\{\left(x_{1}, \ldots, x_{s}\right) \in k^{s} \mid\left(x_{1}^{p}, \ldots, x_{s}^{p}\right) A=\left(x_{1}, \ldots, x_{s}\right)\right\}$; the $\mathbb{F}_{p}$-linear space $L_{0}$ has dimension $s$ because the corresponding equations determine an etale algebra of rank $p^{s}$ over algebraically closed field $k$. The Lemma is proved.

Now suppose for $i=1,2, \mathcal{M}_{i}=\left(M_{i}^{0}, M_{i}^{1}, \varphi_{1}\right)$ and $\mathcal{N}_{i}=\left(N_{i}^{0}, N_{i}^{1}, \varphi_{1}\right)$. Let a vector $\bar{m}_{1} \in\left(M_{1}^{0}\right)^{s}$ and a matrix $C \in M_{s}(S)$ be such that the coordinates of $\bar{m}_{1}$ and $\bar{m}_{1} C$ form an $S$-basis of $M_{1}^{0}$ and, resp., $M_{1}^{1}$, and $\varphi_{1}\left(\bar{m}_{1} C\right)=\bar{m}_{1}$.

If $\bar{n}_{2}=\left(h \circ \theta_{1}\right) \bar{m}_{1}$ then $\varphi_{1}\left(\bar{n}_{2} C\right)=\bar{n}_{2}$. Choose a vector $\bar{m}_{2} \in\left(M_{1}^{0}\right)^{s}$ such that $\theta_{2}\left(\bar{m}_{2}\right)=\bar{n}_{2}$. Then $\bar{m}_{2}-\varphi_{1}\left(\bar{m}_{2} C\right)=\bar{t}_{2} \in T_{2}^{s}$, where $T_{2}=$ 
$\operatorname{Ker} \theta_{2}$. The elements of $\mathcal{L}(h)$ correspond to vectors $\bar{t} \in T_{2}^{s}$ such that $\bar{m}_{2}+\bar{t}=\varphi_{1}\left(\left(\bar{m}_{2}+\bar{t}\right) C\right)$ or equivalently $\bar{t}-\varphi_{1}(\bar{t} C)=-\bar{t}_{2}$.

By above Lemma such $\bar{t}$ always exists and, therefore, $\mathcal{L}(h) \neq \emptyset$. If $\theta_{2}$ is nilpotent then such $\bar{t}$ is unique and part a) is proved.

Now suppose $\theta_{2}$ is special. Then $T_{2}=T_{2}^{\prime}+\left(T_{2} \cap t^{2 e} M_{2}^{0, e t}\right)$ and $\left.\varphi_{1}\right|_{T_{2}^{\prime}}$ is topologically nilpotent. Clearly, we can assume that $T_{2} \cap t^{2 e+1} M_{2}^{0, e t} \subset$ $T_{2}^{\prime}$.

Note that $\mathcal{N}_{2}=\left(M_{2}^{0} / T_{2}, M_{2}^{1} / T_{2}, \varphi_{1}\right)$ and $\theta_{2}$ appears as the composition of two natural projections in $\mathcal{M F}_{S}$

$$
\mathcal{M}_{2} \stackrel{\alpha}{\longrightarrow} \mathcal{N}_{2}^{\prime}:=\left(M_{2}^{0} / T_{2}^{\prime}, M_{2}^{1} / T_{2}^{\prime}, \varphi_{1}\right) \stackrel{\beta}{\longrightarrow} \mathcal{N}_{2} .
$$

Here $\alpha$ is a nilpotent $\varphi_{1}$-lift and, therefore, $f \in \mathcal{L}(h)$ is uniquely determined by $\alpha \circ f \in \operatorname{Hom}_{\mathcal{M F}_{S}}\left(\mathcal{M}_{1}, \mathcal{N}_{2}^{\prime}\right)$.

Suppose for $i=1,2, f_{i} \in \mathcal{L}(h)$ and $f_{i}^{\prime}=\alpha \circ f_{i}$. Then $f_{1}^{\prime}-$ $f_{2}^{\prime} \in \operatorname{Ker} \beta_{*} \subset \operatorname{Hom}_{\mathcal{M F}_{S}}\left(\mathcal{M}_{1}, \widetilde{\mathcal{N}}_{2}\right)$, where $\tilde{\mathcal{N}}_{2}=\left(\widetilde{N}_{2}, \widetilde{N}_{2}, \varphi_{1}\right), \widetilde{N}_{2}=$ $t^{2 e} M_{2}^{0, e t} / t^{2 e+1}$ and $\varphi_{1}$ is $\sigma$-linear automorphism of $\widetilde{N}_{2}$.

Let $\widetilde{\mathcal{M}}_{2}:=\left(t^{2 e} M_{2}^{0, e t}, t^{2 e} M_{2}^{0, e t}, \varphi_{1}\right)$. Clearly it is a subobject of $\mathcal{M}_{2}^{e t}$ in $\mathrm{MF}_{S}^{e}$. Then the natural projection $\widetilde{\mathcal{M}}_{2} \longrightarrow \widetilde{\mathcal{N}}_{2}$ is a nilpotent $\varphi_{1^{-}}$ lift and, therefore, $f_{1}-f_{2}$ factors through the embedding $\widetilde{\mathcal{M}}_{2} \subset \mathcal{M}_{2}^{e t}$. Finally, $\widetilde{\mathcal{M}}_{2}$ is a multiplicative object in $\mathrm{MF}_{S}^{e}$ and this implies that $f_{1}-f_{2}$ factors through the natural projection $\mathcal{M}_{1} \longrightarrow \mathcal{M}_{1}^{\text {mult }}$.

The proposition is proved.

Corollary 1.3. a) If $\theta$ is nilpotent then $\mathcal{M}$ is defined uniquely by $\mathcal{N}$ up to a unique isomorphism in the category $\mathrm{MF}_{S}^{e}$;

b) If $\theta$ is special then $\mathcal{M}$ is defined uniquely by $\mathcal{N}$ up to a unique isomorphism in the category $\mathrm{MF}_{S}^{e *}$.

1.2. Extension of scalars. Let $S^{\prime}=S\left[t^{\prime}\right]$, where $t^{\prime 2}=t$. If $\mathcal{M}=$ $\left(M^{0}, M^{1}, \varphi_{1}\right) \in \mathrm{MF}_{S}^{e}$ then $\mathcal{M} \otimes_{S} S^{\prime}:=\left(M^{0} \otimes_{S} S^{\prime}, M^{1} \otimes_{S} S^{\prime}, \varphi_{1} \otimes \sigma\right) \in$ $\mathrm{MF}_{S^{\prime}}^{2 e}$. (Here $\sigma(s)=s^{2}$ for any $s \in S^{\prime}$.)

If $\mathcal{M}^{\prime}=\left(M^{\prime 0}, M^{\prime 1}, \varphi_{1}^{\prime}\right) \in \mathrm{MF}_{S^{\prime}}^{2 e}$ then $\varphi_{1}^{\prime}\left(M^{\prime 1}\right)=\left\{\varphi_{1}^{\prime}(m) \mid m \in M^{\prime 0}\right\}$ has a natural structure of $S$-module and coincides with $M^{0}$ if $\mathcal{M}^{\prime}=$ $\mathcal{M} \otimes_{S} S^{\prime}$. This fact implies easily the following criterion of the existence of a descent of $\mathcal{M}^{\prime}$ to $S$.

Proposition 1.4. If $\mathcal{M}^{\prime}=\left(M^{\prime 0}, M^{\prime 1}, \varphi_{1}^{\prime}\right) \in \mathrm{MF}_{S^{\prime}}^{2 e}$ then the following two properties are equivalent:

a) $\mathcal{M}=\left(M^{0}, M^{1}, \varphi_{1}\right) \in \mathrm{MF}_{S}^{e}$ is such that $\mathcal{M}^{\prime}=\mathcal{M} \otimes_{S} S^{\prime}$;

b) if $M^{0}=\varphi_{1}^{\prime}\left(M^{1}\right), M^{1}=M^{\prime 1} \cap M^{0}$ and $\varphi_{1}=\left.\varphi_{1}^{\prime}\right|_{M^{1}}$ then $\mathcal{M}=\left(M^{0}, M^{1}, \varphi_{1}\right) \in \mathrm{MF}_{S}^{e}$. 
1.3. The category of augmented $O$-algebras $\operatorname{Aug}_{O}$. Let $\operatorname{Aug}_{O}$ be the category of augmented $O$-algebras $\mathcal{B}=\left(B, I_{B}\right)$ such that $B$ is a flat $O$-algebra of finite rank and $I_{B}$ is an ideal of $B$ such that $O \simeq B / I_{B}$ via the natural map $o \mapsto o \cdot 1_{B}, o \in O$.

We shall denote by $\mathcal{B}^{e t}$ the subobject $\left(B^{e t}, I_{B^{e t}}\right) \in \operatorname{Aug}_{O}$ such that $B^{e t}$ is the maximal etale subalgebra of $B$ and $I_{B^{e t}}=B^{e t} \cap I_{B}$.

With above notation let $I_{B}^{\text {loc }}$ be the ideal of topologically nilpotent elements of $I_{B}$. Clearly, $I_{B}^{\text {loc }} \cap I_{B^{e t}}=\pi I_{B^{e t}}$. Denote by $I_{B}(2)$ the ideal of all $b \in I_{B}$ such that $b^{2} \in 2 I_{B}$ and by $I_{B}(2)^{l o c}$ - the ideal of all $b \in I_{B}$ such that $b^{2} \in 2 I_{B}^{\text {loc }}$. Clearly, $I_{B}(2)=I_{B}(2)^{l o c}+\pi^{e} I_{B^{e t}}$.

Let $J_{B}=I_{B}(2)^{2}+\pi^{e} I_{B}(2)$. Then $J_{B}=\widetilde{J}_{B}+2 I_{B^{e t}}$, where $\widetilde{J}_{B}=$ $I_{B}(2)^{l o c} I_{B}(2)+\pi^{e} I_{B}(2)^{l o c}$. Notice that $J_{B}$ is provided with the standard $D P$-structure by the map $b \mapsto-b^{2} / 2, b \in J_{B}$, and $\widetilde{J}_{B}$ is the maximal ideal in $J_{B}$ where this $D P$-structure is topologically nilpotent.

We shall denote by $\operatorname{Aug}_{O}^{*}$ the following category. Its objects are the objects of the category $\operatorname{Aug}_{O}$ and for any $\mathcal{B}_{1}, \mathcal{B}_{2} \in \operatorname{Aug}_{O}$,

$$
\operatorname{Hom}_{\text {Aug }_{O}^{*}}\left(\mathcal{B}_{1}, \mathcal{B}_{2}\right)=\operatorname{Hom}_{\text {Aug }_{O}}\left(\mathcal{B}_{1}, \mathcal{B}_{2}\right) / R,
$$

where $R$ is the following equivalence relation:

$$
\text { if } f_{1}, f_{2} \in \operatorname{Hom}_{\operatorname{Aug}_{O}}\left(\mathcal{B}_{1}, \mathcal{B}_{2}\right) \text { then } f_{1} \underset{R}{\sim} f_{2} \text { iff } f_{1} \equiv f_{2} \bmod J_{B_{2}} \text {. }
$$

1.4. Families of augmented $O$-algebras $\operatorname{Aug}_{O}(\mathcal{M}), \mathcal{M} \in \mathrm{MF}_{S}^{e}$. Suppose $\mathcal{M}=\left(M^{0}, M^{1}, \varphi_{1}\right) \in \mathrm{MF}_{S}^{e}$ and the vector $\bar{m}^{1}=\left(m_{1}^{1}, \ldots, m_{u}^{1}\right)$ is such that its coordinates form an $S$-basis of $M^{1}$. One can verify that $\bar{m}^{1}$ can be chosen in such way that the following two conditions $C 1$ and $C 2$ are satisfied:

$C 1$ : the non-zero images of all $m_{i}^{1}, 1 \leq i \leq u$, in $M^{0} / t M^{0}$ are linearly independent over $k$;

$C 2: \bar{m}^{1}=\left(\bar{m}^{1, l o c}, t^{e} \bar{m}^{e t}\right)$ where

a) the coordinates of $\bar{m}^{\text {et }}$ form an $S$-basis of $M^{0, e t}$ and $\varphi_{1}\left(t^{e} \bar{m}^{e t}\right)=\bar{m}^{e t}$; b) if $\bar{m}^{l o c}=\varphi_{1}\left(\bar{m}^{1, l o c}\right)$ then the coordinates of $\bar{m}^{l o c} \bmod t$ form a basis of $\bar{M}^{0, l o c}=M^{0, l o c} \otimes_{S} k$ over $k$.

Let $\bar{m}^{0}=\left(\bar{m}^{l o c}, \bar{m}^{e t}\right)$. Then the coordinates of $\bar{m}^{0}$ form an $S$-basis of $M^{0}$. Denote by $U$ the $(n \times n)$-matrix with coefficients in $S$ such that $\bar{m}^{1}=\bar{m}^{0} U$. By condition $C 1$, for appropriate $S$-matrices $U_{1}$ and $U_{2}$, we have $\bar{m}^{1, l o c}=\bar{m}^{l o c} U_{1}+\bar{m}^{e t}\left(t U_{2}\right)$.

The above chosen data: the vectors $\bar{m}^{0}, \bar{m}^{1}$ and the matrix $U \in$ $M_{u}(S)$ - completely describe the structure of $\mathcal{M} \in \mathrm{MF}_{S}^{e}$. Choose $C \in$ $M_{u}(O)$ such that $C \bmod 2=U \bmod t^{2 e}$ with respect to the identification $\kappa_{S O}$. Define the $O$-algebra $A=O[\bar{X}] / \mathcal{I}_{A}$, where $\bar{X}=\left(X_{1}, \ldots, X_{u}\right)$, $\mathcal{I}_{A}=\mathcal{I}_{A, K} \cap O[\bar{X}]$ and $\mathcal{I}_{A, K}$ is the ideal in $K[\bar{X}]$ generated by the 
coordinates of the vector $(-1 / 2)(\bar{X} C)^{(2)}-\bar{X}$. (For any matrix $\alpha=$ $\left(\alpha_{i j}\right)$ we set $\alpha^{(2)}:=\left(\alpha_{i j}^{2}\right)$.)

Proposition 1.5. With above notation $A$ is a flat algebra of rank $2^{n}$ over $O$.

Proof. Indeed, it can be deduced from condition $C 1$ (similarly to Lemma 2.2.2 from [4]) that $C^{(2)}$ divides the scalar matrix $2 I_{u}$ in $M_{u}(O)$. (Note that $C$ divides $\pi^{e} I_{u}$.) This implies that the ideal $\mathcal{I}_{A}$ is generated by the coordinates of the vector $\bar{X}^{(2)}-2(\bar{X}+\bar{V}) C^{(2)^{-1}}$, where $\bar{V}$ consists of $O$-linear combinations of $X_{i} X_{j}, 1 \leqslant i<j \leqslant u$. Therefore, there is an isomorphism of $O$-modules

$$
A \simeq \oplus_{0 \leqslant i_{1}, \ldots, i_{u} \leq 1} O X_{1}^{i_{1}} \ldots X_{u}^{i_{u}}
$$

and $A$ is flat over $O$.

For the above introduced algebra $A$, denote by $I_{A}$ the ideal in $A$ generated by the images of $X_{1}, \ldots, X_{u}$. Then $\left(A, I_{A}\right) \in \operatorname{Aug}_{O}$.

Definition. Denote by $\operatorname{Aug}_{O}(\mathcal{M})$ the family of all augmented algebras $\left(A, I_{A}\right) \in \operatorname{Aug}_{O}$ obtained via the above procedure for all choices of $\bar{m}^{1}$ (which satisfy the conditions $C 1$ and $C 2$ ) and the corresponding matrix $C \in M_{u}(O)$.

1.5. The $\varphi_{1}$-lift $\iota_{\mathcal{M}}$. Define the functor $\iota: \operatorname{Aug}_{O} \longrightarrow \mathcal{M} \mathcal{F}_{S}$ via

$$
\left(B, I_{B}\right) \mapsto\left(I_{B} / J_{B}, I_{B}(2) / J_{B}, \varphi_{1}\right)
$$

where $\left(B, I_{B}\right) \in \operatorname{Aug}_{O}, J_{B}$ was introduced in Subsection 1.3 and $\varphi_{1}$ is induced by the correspondences $b \mapsto-b^{2} / 2, b \in I_{B}$.

For any $\mathcal{M} \in \mathrm{MF}_{S}^{e}$ and $\left(A, I_{A}\right) \in \operatorname{Aug}_{O}(\mathcal{M})$, there is a canonical morphism $\iota_{\mathcal{M}}: \mathcal{M} \longrightarrow \iota(A(\mathcal{M}))$ in $\mathcal{M F}_{S}$ such that $\bar{m}^{0} \mapsto \bar{X} \bmod J_{A}$ and $\bar{m}^{1} \mapsto \bar{X} C \bmod J_{A}$. Clearly, the image $\iota_{\mathcal{M}}(\mathcal{M})$ is a subobject of $\iota(\mathcal{A}(\mathcal{M}))$ in the category $\mathcal{M F}_{S}$.

Proposition 1.6. The map $\iota_{\mathcal{M}}: \mathcal{M} \longrightarrow \iota_{\mathcal{M}}(\mathcal{M})$ is a special $\varphi_{1}$-lift.

Proof. Consider $\widetilde{\mathcal{M}}=\mathcal{M} \otimes_{S} S / t^{2 e} \in \mathcal{M F}_{S}$. Clearly, the natural projection $\mathcal{M} \longrightarrow \widetilde{\mathcal{M}}$ is a special $\varphi_{1}$-lift and $\iota_{\mathcal{M}}$ is the composition of this projection and a unique $\tilde{\iota}_{\mathcal{M}} \in \operatorname{Hom}_{\mathcal{M} \mathcal{F}_{S}}\left(\widetilde{\mathcal{M}}, \iota_{\mathcal{M}}(\mathcal{M})\right)$.

Let $\widetilde{\mathcal{M}}=\left(\widetilde{M}^{0}, \widetilde{M}^{1}, \varphi_{1}\right)$. Then

$$
\begin{gathered}
\widetilde{M}^{0}=\left\{\sum o_{i} \widetilde{X}_{i} \mid o_{1}, \ldots, o_{n} \in O, \widetilde{X}_{i}=X_{i} \bmod 2 I_{A}\right\} \\
\widetilde{M}^{1}=\left\{\sum o_{i} \widetilde{Y}_{i} \mid o_{1}, \ldots, o_{n} \in O,\left(\widetilde{Y}_{1}, \ldots, \widetilde{Y}_{n}\right)=\left(\widetilde{X}_{1}, \ldots, \widetilde{X}_{n}\right) C\right\}
\end{gathered}
$$

where the $S$-module structure is induced by the given $O$-module structure via the identification $\kappa_{S O}$ and $\varphi_{1}: \widetilde{M}^{1} \longrightarrow \widetilde{M}^{0}$ is given via the correspondence $\sum o_{i} \widetilde{Y}_{i} \mapsto \sum o_{i}^{2} \widetilde{X}_{i}$. 
Suppose $\tilde{\iota}_{\mathcal{M}}=\left(\tilde{\iota}_{\mathcal{M}}^{0}, \tilde{\iota}_{\mathcal{M}}^{1}\right)$. Then

$$
\begin{aligned}
\widetilde{T}^{0} & :=\operatorname{Ker} \tilde{\iota}_{\mathcal{M}}^{0}=\left\{\sum o_{i} \widetilde{X}_{i} \mid \sum o_{i} X_{i} \in J_{A}\right\} \\
\widetilde{T}^{1} & :=\operatorname{Ker} \tilde{\iota}_{\mathcal{M}}^{1}=\left\{\sum o_{i} \widetilde{Y}_{i} \mid \sum o_{i} Y_{i} \in J_{A}\right\}
\end{aligned}
$$

The proposition will be proved if we show $\widetilde{T}^{0}=\widetilde{T}^{1}, \varphi_{1}\left(\widetilde{T}^{0}\right) \subset \widetilde{T}^{0}$ and $\left.\varphi_{1}\right|_{\widetilde{T}^{0}}$ is nilpotent.

Suppose $\tilde{v}=\sum o_{i} \widetilde{X}_{i} \in \widetilde{T}^{0}$. Then $\sum o_{i} X_{i} \in J_{A} \subset I_{A}(2)$ and $\sum_{i} o_{i}^{2} X_{i}^{2} \in 2 I_{A}$. Let $\left(G_{1}^{\prime}, \ldots, G_{u}^{\prime}\right)=2(\bar{X}+\bar{V})\left(C^{(2)}\right)^{-1}$, cf. the proof of Proposition 1.5. Then $\sum_{i} o_{i}^{2} G_{i}^{\prime} \in 2 I_{A}$ and due to the isomorphism (1.1) we can follow the linear terms to obtain that

$$
2\left(o_{1}^{2}, \ldots, o_{u}^{2}\right)\left(C^{(2)}\right)^{-1}:=2\left(\alpha_{1}, \ldots, \alpha_{n}\right) \in 2 O^{u}
$$

Clearly, there are $\alpha_{1}^{\prime}, \ldots, \alpha_{u}^{\prime} \in O$ such that all $\alpha_{i}^{\prime 2} \equiv \alpha_{i} \bmod 2 O$ and (1.2) implies that

$$
\left(o_{1}, \ldots, o_{u}\right) \equiv\left(\alpha_{1}^{\prime}, \ldots, \alpha_{u}^{\prime}\right) C \bmod \pi^{e} .
$$

Therefore, $\sum_{i} o_{i} \widetilde{X}_{i}$ is congruent modulo $\pi^{e} \widetilde{M}^{0}$ to an $O$-linear combination of the coordinates of the vector $\left(\widetilde{Y}_{1}, \ldots, \widetilde{Y}_{u}\right)=\left(\widetilde{X}_{1}, \ldots, \widetilde{X}_{u}\right) C$. In other words, $\tilde{v} \in \widetilde{M}^{1}$, i.e. $\widetilde{T}^{0}=\widetilde{T}^{1}$.

If $\tilde{v}=\sum o_{i}^{\prime} \widetilde{Y}_{i}$, then

$$
\varphi_{1}(\widetilde{v})=\sum o_{i}^{\prime 2} \widetilde{X}_{i}=-\widetilde{v}^{2} / 2+\sum_{i, j} o_{i}^{\prime} o_{j}^{\prime} \widetilde{Y}_{i} \widetilde{Y}_{j} \in J_{A} \bmod 2 I_{A}
$$

implies $\varphi_{1}\left(\widetilde{T}^{0}\right) \subset \widetilde{T}^{0}$. (Use that $J_{A}$ is a $D P$-ideal and $I_{A}(2)^{2} \subset J_{A}$.)

Finally, let $\tilde{v}_{0}=\tilde{v}$ and for $n \geqslant 0, \tilde{v}_{n+1}=\varphi_{1}\left(\tilde{v}_{n}\right)$. We must prove that for $n \gg 0, \tilde{v}_{n}=0$.

Let $A^{\prime}=O\left[Y_{1}, \ldots, Y_{n}\right]$. Then $A^{\prime}$ is an $O$-subalgebra in $A$ given by the equations

$$
\left(Y_{1}^{2}, \ldots, Y_{n}^{2}\right)+\left(Y_{1}, \ldots, Y_{n}\right)\left(2 C^{-1}\right)=0 .
$$

Therefore, any element $a \in A^{\prime}$ can be uniquely presented in the form

$$
a=\sum_{1 \leqslant i_{1}<\cdots<i_{s} \leqslant u} o_{i_{1} \ldots i_{s}}(a) Y_{i_{1}} \ldots Y_{i_{s}},
$$

where all $o_{i_{1} \ldots i_{s}}(a) \in O$. Set $\mathcal{L}(a)=o_{1}(a) Y_{1}+\cdots+o_{u}(a) Y_{u}$. Notice that if $I_{A^{\prime}}$ is the augmentation ideal of $A^{\prime}$ generated by $Y_{1}, \ldots, Y_{u}$ and $a \in I_{A^{\prime}}^{2}$ then all $o_{i}(a) \equiv 0 \bmod \pi^{e}$ (use that $\left.2 C^{-1} \equiv 0 \bmod \pi^{e}\right)$. This means that if $a_{1}, a_{2} \in I_{A^{\prime}}$ and $a_{1} \equiv a_{2} \bmod I_{A^{\prime}}^{2}$ then $\mathcal{L}\left(a_{1}\right) \equiv \mathcal{L}\left(a_{2}\right) \bmod \pi^{e} I_{A^{\prime}}$.

With above notation let $v_{0}=\sum o_{i} X_{i}$ and for all $n \geqslant 0, v_{n+1}=$ $-v_{n}^{2} / 2$. Clearly, all $v_{n} \in J_{A}$ and there is an $N_{0} \geqslant 0$ such that $v_{N_{0}} \in 2 I_{A}$.

For $n \geqslant 0$, set $v_{n}^{*}=\mathcal{L}\left(v_{n}\right)$ and denote by $\rho: A^{\prime} \longrightarrow A / 2$ the composition of the natural inclusion $A^{\prime}$ into $A$ and the reduction map $A \longrightarrow A / 2$.

Lemma 1.7. $\rho\left(v_{n}^{*}\right) \equiv \tilde{v}_{n} \bmod \left(\pi^{e} \widetilde{M}^{1}\right)$. 
Proof of Lemma. Use induction on $n \geqslant 0$.

Clearly, $v_{0}=v_{0}^{*}$ and $\rho\left(v_{0}\right)=\tilde{v}_{0}$.

Suppose $\rho\left(v_{n}^{*}\right) \equiv \tilde{v}_{n} \bmod \left(\pi^{e} \widetilde{M}^{1}\right)$.

If $v_{n}^{*}=\sum o_{i}^{(n)} Y_{i}$, where all $o_{i}^{(n)} \in O$, then $v_{n}=\sum o_{i}^{(n)} Y_{i}+\alpha$ with $\alpha \in I_{A^{\prime}}^{2}$. Therefore,

$$
\begin{gathered}
v_{n+1} \equiv-v_{n}^{2} / 2 \equiv \sum o_{i}^{(n) 2} X_{i} \bmod I_{A^{\prime}}^{2} \\
v_{n+1}^{*} \equiv \sum o_{i}^{(n) 2} X_{i} \bmod \pi^{e} I_{A^{\prime}}
\end{gathered}
$$

and $\rho\left(v_{n+1}^{*}\right) \equiv \varphi_{1}\left(\rho\left(v_{n}^{*}\right)\right) \equiv \varphi_{1}\left(\tilde{v}_{n}\right) \bmod \left(\pi^{e} \widetilde{M}^{1}\right)$. The lemma is proved.

Finally, the above lemma implies that $\tilde{v}_{N_{0}} \in \pi^{e} \widetilde{M}^{1}$ and, therefore, $\tilde{v}_{N_{0}+1}=\varphi_{1}\left(\tilde{v}_{N_{0}}\right) \in \pi^{2 e} \widetilde{M}^{0}=0$. The proposition is proved.

1.6. The maps $\Theta^{*}$ and $\Psi^{*}$. Suppose $\mathcal{B}=\left(B, I_{B}\right) \in \operatorname{Aug}_{O}$ and $\mathcal{B}^{e t}=$ $\left(B^{e t}, I_{B^{e t}}\right)$ is the maximal etale subalgebra in $\mathcal{B}$. Then $J_{B^{e t}}=2 I_{B^{e t}}$ and $\iota\left(\mathcal{B}^{e t}\right)=\left(I_{B^{e t}} / 2 I_{B^{e t}}, \pi^{e} I_{B^{e t}} / 2 I_{B^{e t}}, \varphi_{1}\right) \in \mathcal{M} \mathcal{F}_{S}$ admits a (unique) special $\varphi_{1}$-lift $\mathcal{E}\left(\mathcal{B}^{e t}\right) \in \mathrm{MF}_{S}^{e, e t}$.

Introduce $\mathrm{m}\left(\mathcal{B}^{e t}\right)=\left(2 I_{B^{e t}} / 2 \pi I_{B^{e t}}, 2 I_{B^{e t}} / 2 \pi I_{B^{e t}}, \varphi_{1}\right) \in \mathcal{M} \mathcal{F}_{S}$, where $\varphi_{1}$ is induced (as usually) by the map $a \mapsto-a^{2} / 2, a \in 2 I_{B^{e t}}$. Clearly, $\mathrm{m}\left(\mathcal{B}^{e t}\right)$ admits a (unique) nilpotent $\varphi_{1}$-lift $M\left(\mathcal{B}^{e t}\right) \in \mathrm{MF}_{S}^{e, m u l t}$ and the identity morphism on $I_{B^{e t}}$ induces the natural morphism

$$
\omega\left(\mathcal{B}^{e t}\right): M\left(\mathcal{B}^{e t}\right) \longrightarrow \mathcal{E}\left(\mathcal{B}^{e t}\right)
$$

in the category $\mathrm{MF}_{S}^{e}$.

Suppose $\mathcal{M}=\left(M^{0}, M^{1}, \varphi_{1}\right) \in \operatorname{MF}_{S}^{e}$ and $\mathcal{A}=\left(A, I_{A}\right) \in \operatorname{Aug}_{O}(\mathcal{M})$. Introduce the map

$$
\Theta: \operatorname{Hom}_{\text {Aug }_{O}}(\mathcal{A}, \mathcal{B}) \longrightarrow \operatorname{Hom}_{\mathcal{M F}_{S}}(\mathcal{M}, \iota(\mathcal{B}))
$$

by attaching to $F \in \operatorname{Hom}_{\text {uug }_{O}}(\mathcal{A}, \mathcal{B})$ the morphism of filtered modules $\Theta(F)=\iota(F) \circ \iota_{\mathcal{M}}$.

Proposition 1.8. With the above notation:

a) $\Theta$ is surjective;

b) if either $\mathcal{B}^{\text {et }}=\mathcal{S}^{\text {et }}$ or $\mathcal{M}^{\text {mult }}=0$ then $\theta$ is bijective;

c) there is a natural strict action of the group $\operatorname{Hom}_{\mathrm{MF}_{S}^{e}}\left(\mathcal{M}^{\text {mult }}, \mathcal{E}\left(\mathcal{B}^{e t}\right)\right)$ on $\operatorname{Hom}_{\text {Augo }_{o}}(\mathcal{A}, \mathcal{B})$ and the corresponding equivalence relation $R$ coincides with the equivalence relation from the definition of $\operatorname{Aug}_{O}^{*}$ in Subsection 1.3;

d) $\Theta$ induces the bijection

$$
\Theta^{*}: \operatorname{Hom}_{\operatorname{Aug}_{O}^{*}}(\mathcal{A}, \mathcal{B}) \longrightarrow \operatorname{Hom}_{\mathcal{M} \mathcal{F}_{S}}\left(\iota_{\mathcal{M}}(\mathcal{M}), \iota(\mathcal{B})\right) .
$$

Proof. Suppose $\mathcal{A}=\left(A, I_{A}\right) \in \operatorname{Aug}_{O}(\mathcal{M})$ is given via a special choice of vectors $\bar{m}^{0}$ and $\bar{m}^{1}$ with the coordinates in $M^{0}$ and, resp., $M^{1}$, and the matrix $C \in M_{u}(O)$ from Subsection 1.4. 
Lemma 1.9. Suppose $\bar{b}^{0} \in I_{B}^{u}$ is such that $(-1 / 2)\left(\bar{b}^{0} C\right)^{(2)} \equiv \bar{b}^{0} \bmod J_{B}$. Let $\mathcal{L}\left(\bar{b}^{0}\right)$ be the set of all $\bar{b} \in I_{B}^{u}$ such that $\bar{b} \equiv \bar{b}^{0} \bmod \widetilde{J}_{B}$ and it holds $(-1 / 2)(\bar{b} C)^{(2)} \equiv \bar{b} \bmod \widetilde{J}_{B}$. Then

a) $\mathcal{L}\left(\bar{b}^{0}\right) \neq \emptyset$;

b) if $\bar{b}_{1}, \bar{b}_{2} \in \mathcal{L}\left(\bar{b}^{0}\right)$ then $\bar{x}=\bar{b}_{1}-\bar{b}_{2} \in J_{B}^{u}$ and $(-1 / 2)(\bar{x} C)^{(2)} \equiv$ $\bar{x} \bmod \widetilde{J}_{B}$.

Proof of Lemma. The vector $\bar{b}=\bar{b}^{0}+\bar{x} \in \mathcal{L}\left(\bar{b}^{0}\right)$ iff $\bar{x} \in J_{B}^{u}$ and

$$
(-1 / 2)(\bar{x} C)^{(2)}-\bar{x} \equiv \bar{b}^{0}+(1 / 2)\left(\bar{b}^{0} C\right)^{(2)} \bmod \widetilde{J}_{B} .
$$

Notice that $\mathcal{V}=\left(J_{B} / \widetilde{J}_{B}\right)^{n}$ has a natural structure of a finite dimensional vector space over $k$ and the correspondence $\bar{x} \mapsto(-1 / 2)(\bar{x} C)^{(2)}$ induces a $\sigma$-linear morphism $\tilde{\varphi}: \mathcal{V} \longrightarrow \mathcal{V}$. By Lemma 1.2, $\tilde{\varphi}-$ id : $\mathcal{V} \longrightarrow \mathcal{V}$ is surjective. This proves part a). Part b) follows easily from the congruence $\left(\bar{b}_{1} C\right)^{(2)} \equiv\left(\bar{b}_{2} C\right)^{(2)}+(\bar{x} C)^{(2)} \bmod 2 J_{B} I_{B}(2)$.

Notice that $\operatorname{Hom}_{\mathcal{M F}_{S}}\left(\iota_{\mathcal{M}}(\mathcal{M}), \iota(\mathcal{B})\right)=$

$$
\begin{gathered}
\left\{\bar{b}^{0} \bmod J_{B} \mid \bar{b}^{0} \in I_{B}^{u},(-1 / 2)\left(\bar{b}^{0} C\right)^{(2)} \equiv \bar{b}^{0} \bmod J_{B}\right\}, \\
\operatorname{Hom}_{\text {Augo }_{O}}(\mathcal{A}, \mathcal{B})=\left\{\bar{b} \in I_{B}^{u} \mid(-1 / 2)(\bar{b} C)^{(2)}=\bar{b}\right\} \\
=\left\{\bar{b} \bmod \widetilde{J}_{B} \mid \bar{b} \in I_{B}^{u},(-1 / 2)(\bar{b} C)^{(2)} \equiv \bar{b} \bmod \widetilde{J}_{B}\right\}
\end{gathered}
$$

and the map $\Theta$ is given via $\bar{b} \bmod \widetilde{J}_{B} \mapsto \bar{b}^{0} \bmod J_{B}$.

Therefore, part a) of Proposition 1.8 follows from part a) of above Lemma. If $\bar{x}$ is the vector from part b) of above Lemma then the correspondence $\bar{m} \mapsto \bar{x}$ identifies

$$
\operatorname{Hom}_{\mathrm{MF}_{S}^{e}}\left(\mathcal{M}, M\left(\mathcal{B}^{e t}\right)\right)=\operatorname{Hom}_{\mathcal{M} \mathcal{F}_{S}}\left(\mathcal{M}^{\text {mult }}, M\left(\mathcal{B}^{e t}\right)\right)
$$

with $\operatorname{Hom}_{\mathcal{M F}_{S}}\left(\mathcal{M}^{\text {mult }}, \mathrm{m}\left(\mathcal{B}^{\text {et }}\right)\right)$. This implies part b) of Proposition 1.8. With the above notation the correspondence $\bar{b} \mapsto \bar{b}+\bar{x}$ determines the action of $\operatorname{Hom}_{\mathrm{MF}_{S}^{e}}\left(\mathcal{M}^{\text {mult }}, \mathcal{E}\left(\mathcal{B}^{e t}\right)\right)$ on $\operatorname{Hom}_{\text {Augo }}(\mathcal{A}, \mathcal{B})$. One can easily verify that this action is strict and $\Theta$ induces bijection of the corresponding quotient $\operatorname{Hom}_{\mathrm{Aug}_{\mathcal{O}}}(\mathcal{A}, \mathcal{B}) / R$ and $\operatorname{Hom}_{\mathcal{M} \mathcal{F}_{S}}\left(\iota_{\mathcal{M}}(\mathcal{M}), \iota(\mathcal{B})\right)$.

Remark. a) By condition $C 2, \bar{m}^{0}=\left(\bar{m}^{l o c}, \bar{m}^{e t}\right)$ and therefore we have the appropriate presentation $\bar{x}=\left(\bar{x}^{l o c}, \bar{x}^{e t}\right)$, where $\bar{x}$ is the vector from part b) of Lemma 1.9. One can easily see that $\bar{x}^{e t}=\overline{0}$. In particular, the shifts by all above vectors $\bar{x}$ determine a strict action of $\operatorname{Hom}_{\mathcal{M F}_{\mathcal{S}}}\left(\mathcal{M}^{\text {mult }}, \iota(\mathcal{B})\right)$ on $I_{B}^{u} \bmod \widetilde{J}_{B}$.

b) One can easily see that if $\mathcal{M} \in \mathrm{MF}_{S}^{\text {e,mult }}$ and $\mathcal{B}=\mathcal{B}^{\text {et }}$ then

$$
\operatorname{Hom}_{\text {Augo }_{O}}(\mathcal{A}, \mathcal{B})=\operatorname{Hom}_{\mathcal{M} \mathcal{F}_{S}}\left(\mathcal{M}, E\left(\mathcal{B}^{e t}\right)\right) ;
$$

Corollary 1.10. If $\mathcal{B} \in \operatorname{Aug}_{O}(\mathcal{N})$ with $\mathcal{N} \in \mathrm{MF}_{S}^{e}$ then the above identification $\Theta^{*}$ induces a functorial in both arguments embedding

$$
\Psi^{*}: \operatorname{Hom}_{\mathrm{MF}_{S}^{*}}(\mathcal{M}, \mathcal{N}) \longrightarrow \operatorname{Hom}_{\text {Augo }_{O}^{*}}(\mathcal{A}, \mathcal{B})
$$


and the correspondences $\mathcal{M} \mapsto \mathcal{A}$ (and $\mathcal{N} \mapsto \mathcal{B}$ ) induce a faithful functor $\Psi^{*}: \mathrm{MF}_{S}^{e *} \longrightarrow \operatorname{Aug}_{O}^{*}$.

1.7. Group schemes $\operatorname{Spec} A,\left(A, I_{A}\right) \in \operatorname{Aug}_{O}(\mathcal{M})$. Suppose $\mathcal{M} \in$ $\operatorname{MF}_{S}^{e}$ and $\mathcal{A}=\left(A, I_{A}\right) \in \operatorname{Aug}_{O}(\mathcal{M})$ is given via a special choice of vectors $\bar{m}^{0}, \bar{m}^{1}$ and matrices $U \in M_{u}(S), C \in M_{u}(O)$ under assumptions $C 1$ and $C 2$ from Subsection 1.4.

We can describe the structures of $\mathcal{M} \oplus \mathcal{M}$ and $A \otimes_{O} A$ via the $S$ basis $\bar{m}^{0} \oplus\{0\},\{0\} \oplus \bar{m}^{0}$ for $M^{0} \oplus M^{0}$, the $S$-basis $\bar{m}^{1} \oplus\{0\},\{0\} \oplus \bar{m}^{1}$ for $M^{1} \oplus M^{1}$ and the corresponding matrices $\left(\begin{array}{cc}U & 0 \\ 0 & U\end{array}\right) \in M_{2 u}(S)$ and $\left(\begin{array}{cc}C & 0 \\ 0 & C\end{array}\right) \in M_{2 u}(O)$. (One can easily see that these data satisfy assumptions $C 1$ and $C 2$ from Subsection 1.4.) Note that $A=$ $O[\bar{X}] / \mathcal{I}_{A}$, where the ideal $\mathcal{I}_{A}$ is generated by the coordinates of the vector $\left((\bar{X} C)^{(2)}+2 \bar{X}\right) C^{(2)^{-1}}$,

$$
\mathcal{A} \otimes \mathcal{A}=\left(A \otimes_{O} A, I_{A} \otimes_{O} A+A \otimes_{O} I_{A}\right) \in \operatorname{Aug}_{O}(\mathcal{M})
$$

and

$$
A \otimes_{O} A=O[\bar{X} \otimes 1,1 \otimes \bar{X}] /\left(\mathcal{I}_{A} \otimes 1,1 \otimes \mathcal{I}_{A}\right) .
$$

Let $e: A \longrightarrow A / I_{A}=O$ be the natural projection and let $\Delta_{\mathcal{A}}^{*}=$ $\Psi^{*}(\nabla) \in \operatorname{Hom}_{\text {Aug }_{O}^{*}}(\mathcal{A}, \mathcal{A} \otimes \mathcal{A})$, where $\nabla: \mathcal{M} \longrightarrow \mathcal{M} \oplus \mathcal{M}$ is the class of the diagonal morphism in the category $\mathrm{MF}_{S}^{e *}$.

Let $\mathcal{L}_{\mathcal{A}}$ be the set of all $\Delta \in \operatorname{Hom}_{\mathrm{Aug}_{O}}(\mathcal{A}, \mathcal{A} \otimes \mathcal{A})$ such that:

- $\Delta \bmod R=\Delta_{\mathcal{A}}^{*}$;

- $G=\operatorname{Spec} A$ becomes an object of the category $\mathrm{Gr}_{O}$ when provided with the counit $e$ and the coaddition $\Delta$.

Proposition 1.11. a) $\mathcal{L}_{\mathcal{A}} \neq \emptyset$;

b) If $\Delta_{1}, \Delta_{2} \in \mathcal{L}_{\mathcal{A}}$ then the corresponding coalgebra structures on $\mathcal{A}$ are transformed one into another via an automorphism $f \in \operatorname{Aug}_{O}(\mathcal{A})$ such that $f \underset{R}{\sim} \operatorname{id}_{\mathcal{A}}$ (i.e. $f$ and $\operatorname{id}_{\mathcal{A}}$ coincide in $\operatorname{Aug}_{O}^{*}$ ).

Proof. Let $\bar{X}=\left(\bar{X}^{l o c}, \bar{X}^{e t}\right)$ with respect to the presentation $\bar{m}=$ $\left(\bar{m}^{l o c}, \bar{m}^{e t}\right)$ from condition $C 2$ in Subsection 1.4. For $\Delta \in \mathcal{L}_{\mathcal{A}}$, set

$$
\Delta(\bar{X})=\left(\Delta\left(\bar{X}^{l o c}\right), \Delta\left(\bar{X}^{e t}\right)\right)=\bar{X} \otimes 1+1 \otimes \bar{X}+\bar{\jmath},
$$

where $\bar{\jmath}=\left(\bar{\jmath}^{o c}, \bar{\jmath}^{e t}\right)$.

Note that $\bar{j}^{\text {et }}$ does not depend on a choice of $\Delta \in \mathcal{L}_{\mathcal{A}}$. This implies that $G^{e t}:=\operatorname{Spec} A^{e t} \in \operatorname{Gr}_{O}^{e t}$ when provided with the coaddition $\Delta^{e t}=$ $\left.\Delta\right|_{A^{e t}}$ and the counit $e^{e t}=\left.e\right|_{A^{e t}}$. More explicitly, $A^{e t}=O\left[\bar{X}^{e t}\right]$ with the equations $\eta \bar{X}^{e t(2)} \equiv \bar{X}^{e t} \bmod 2 I_{A^{e t}}$, where $\eta=-\pi^{2 e} / 2 \in O^{*}$, and $\Delta\left(\bar{X}^{e t}\right) \equiv \bar{X}^{e t} \otimes 1+1 \otimes \bar{X}^{e t} \bmod 2 I_{A^{e t} \otimes A^{e t}}$. 
Let $G_{k}^{e t}:=G^{e t} \otimes_{O} k=\operatorname{Spec} A_{k}^{e t}$. Remind that the $k$-module of symmetric Hochschild 2-cocycles $Z_{\text {sym }}^{2}\left(G_{k}^{e t}\right)$ consists of symmetric $\gamma \in$ $I_{A_{k}^{e t} \otimes A_{k}^{e t}}$ such that

$$
(\Delta \otimes \mathrm{id}) \gamma+\gamma \otimes 1=1 \otimes \gamma+(\mathrm{id} \otimes \Delta) \gamma .
$$

The corresponding $k$-module of 2 -coboundaries equals

$$
B^{2}\left(G_{k}^{e t}\right)=\left\{\Delta^{e t}(\gamma)-\gamma \otimes 1-1 \otimes \gamma \mid \gamma \in I_{A_{k}^{e t}}\right\} \subset Z_{s y m}^{2}\left(G_{k}^{e t}\right)
$$

We have the following two facts:

- Suppose $\gamma \in Z_{\text {sym }}^{2}\left(G_{k}^{e t}\right)$ and mult : $A_{k}^{e t} \otimes A_{k}^{e t} \longrightarrow A_{k}^{e t}$ is the morphism of multiplication. Then $\gamma \in B^{2}\left(G_{k}^{e t}\right) \Leftrightarrow \operatorname{mult}(\gamma)=0$.

- If $\bar{X}^{e t}=\left(X_{1}^{e t}, \ldots, X_{u_{e t}}^{e t}\right)$ then the elements $\delta^{+}\left(X_{i_{1}}^{e t} \ldots X_{i_{s}}^{e t}\right) \bmod \pi$, where $s \geqslant 2,1 \leqslant i_{1}<\cdots<i_{s} \leqslant u_{e t}$ and $\delta^{+}=\Delta-\mathrm{id} \otimes 1-1 \otimes \mathrm{id}$, form a $k$-basis of $B^{2}\left(G_{k}^{e t}\right)$ and the correspondences $\delta^{+}\left(X_{i_{1}}^{e t} \ldots X_{i_{s}}^{e t}\right) \mapsto$ $X_{i_{1}}^{e t} \ldots X_{i_{s}}^{e t}$ determine the $k$-linear embedding $\omega: B^{2}\left(G_{k}^{e t}\right) \longrightarrow I_{A}^{e t} \bmod \pi$. Note that for any $\alpha \in B^{2}\left(G_{k}^{e t}\right), \alpha^{2} \in B^{2}\left(G_{k}^{e t}\right)$ and $\omega\left(\alpha^{2}\right)=\omega(\alpha)^{2}$.

Now notice that $\Delta$ depends only on the residue

$$
\bar{\jmath} \bmod \widetilde{J}_{A \otimes A} \in J_{A \otimes A}^{u} \bmod \widetilde{J}_{A \otimes A}=2 I_{A^{e t} \otimes A^{e t}}^{u \bmod }\left(2 \pi I_{A^{e t} \otimes A^{e t}}\right)
$$

Let $\bar{\jmath}=2 \bar{\alpha} \bmod 2 \pi I_{A^{e t} \otimes A^{e t}}$, where $\bar{\alpha}(\Delta)=\left(\bar{\alpha}^{l o c}(\Delta), \bar{\alpha}^{e t}\right) \in I_{A^{e t} \otimes A^{e t}}^{u}$.

We have the following properties:

a) $\Delta$ defines a morphism of augmented algebras iff $(\bar{\alpha} C)^{(2)}+\bar{\alpha}$ has all its coordinates in $\pi I_{A^{e t} \otimes A^{e t}}$.

b) $\Delta$ determines a structure of commutative group scheme on $G=$ $\operatorname{Spec} A$ iff $\bar{\alpha} \bmod \pi I_{A^{e t} \otimes A^{e t}}$ has all its coordinates in $Z_{\text {sym }}^{2}\left(G_{k}^{e t}\right)$.

c) $2 \operatorname{id}_{G}=0$ iff $\bar{\alpha}^{l o c} \bmod \pi I_{A^{e t} \otimes A^{e t}}$ has all its coordinates in $B^{2}\left(G_{k}^{e t}\right)$.

The proof of property a) uses the equations $(-1 / 2)(\bar{X} C)^{(2)}=\bar{X}$ for $A$, property $\mathrm{b}$ ) is equivalent to the axioms of coassociativity and cocommutativity for $G$. As for property c), note that $\overline{0}=2 \operatorname{id}_{G}(\bar{X})=$ $\operatorname{mult}(\Delta(\bar{X}))=2 \bar{X}+\operatorname{mult}(\bar{\jmath})$ and, therefore, mult ${ }^{l o c}$ has all its coordinates in $\widetilde{J}_{A \otimes A}$ or, equivalently, $\operatorname{mult}\left(\bar{\alpha}^{l o c}\right) \equiv 0 \bmod \pi I_{A^{e t}}$.

Now we can finish the proof of Proposition 1.11.

Let $\Delta_{0} \in \Delta_{\mathcal{A}}^{*}$ be such that

$$
\Delta_{0}\left(\bar{X}^{l o c}\right) \equiv \bar{X}^{l o c} \otimes 1+1 \otimes \bar{X}^{l o c} \bmod \widetilde{J}_{A \otimes A} .
$$

This means that $\bar{\alpha}\left(\Delta_{0}\right)=\left(\overline{0}, \bar{\alpha}^{e t}\right)$ and by above properties a)-c), we have $\Delta_{0} \in \mathcal{L}_{\mathcal{A}}$.

Suppose $\Delta \in \mathcal{L}_{\mathcal{A}}$ and $\bar{\alpha}(\Delta)=\left(\bar{\alpha}^{l o c}(\Delta), \bar{\alpha}^{e t}\right)$. Let $\bar{\gamma}=\left(\gamma^{l o c}, \overline{0}\right) \in I_{A^{e t}}^{n}$ be such that $\delta^{+}\left(\bar{\gamma}^{l o c} \bmod \pi I_{A^{e t}}\right)=\bar{\alpha}^{l o c} \bmod \pi I_{A^{e t} \otimes A^{e t}}$. We can assume that $\bar{\gamma}^{l o c}=\omega\left(\delta^{+} \bar{\alpha}^{l o c}\right)$ and, therefore, $(\bar{\gamma} C)^{(2)}+\bar{\gamma}$ has all its coordinates in $\pi I_{A^{e t}}$. Therefore, there is a unique $F \in \operatorname{Hom}_{\text {Aug }_{O}}(\mathcal{A}, \mathcal{A} \otimes \mathcal{A})$ such that $F(\bar{X}) \equiv \bar{X}+2 \bar{\gamma} \bmod \widetilde{J}_{A \otimes A}$. Clearly, $F \underset{R}{\sim} \operatorname{id}_{\mathcal{A}}$ in $\operatorname{Aug}_{O}^{*}$. 
In addition, $\Delta_{0}(\bar{F}(\bar{X})) \equiv \Delta_{0}(\bar{X}+2 \bar{\gamma}) \equiv$

$$
\begin{gathered}
\left(\bar{X}^{l o c} \otimes 1+1 \otimes \bar{X}^{l o c}+2 \Delta^{e t}\left(\bar{\gamma}^{l o c}\right), \Delta_{0}\left(\bar{X}^{e t}\right)\right) \\
\equiv\left(\bar{X}^{l o c} \otimes 1+1 \otimes \bar{X}^{l o c}+2 \bar{\jmath}^{l o c}+2\left(\bar{\gamma}^{l o c} \otimes 1+1 \otimes \bar{\gamma}^{l o c}\right), \Delta\left(\bar{X}^{e t}\right)\right) \\
\equiv(F \otimes F)(\Delta(\bar{X})) \bmod \widetilde{J}_{A \otimes A} .
\end{gathered}
$$

Therefore, $F \circ \Delta_{0}=\Delta \circ(F \otimes F)$. The Proposition is proved.

\subsection{Functor $\mathcal{G}_{O}$.}

Proposition 1.12. There is a functor $\mathcal{G}_{O}: \mathrm{MF}_{S}^{e *} \longrightarrow \mathrm{Gr}_{O}^{*}$ such that its compositoon with the forgetful functor $\mathrm{Gr}_{O}^{*} \longrightarrow$ Augo $_{O}^{*}$ coincides with the functor $\Psi^{*}$ from Corollary 1.10 .

Proof. For $i=1,2$, let

- $\mathcal{M}_{i} \in \mathrm{MF}_{S}^{e}$ with specially chosen vectors $\bar{m}_{i}^{0}, \bar{m}_{i}^{1}$ satisfying the conditions $C 1, C 2$ fom Subsection 1.4,

- $\mathcal{A}_{i}=\left(A_{i}, I_{A_{i}}\right)$ be the corresponding augmented $O$-algebras with the coalgebra structures uniquely given by the coadditions $\Delta_{i}: A_{i} \longrightarrow$ $A_{i} \otimes A_{i}$ such that $\Delta_{i} \in \Psi^{*}\left(\nabla_{i}\right)$ (where $\nabla_{i}$ are the diagonal maps from

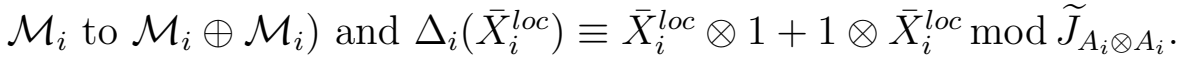

Denote the corresponding group schemes $\operatorname{Spec} A_{i}=G_{i} \in \mathrm{Gr}_{O}$.

Suppose $f \in \operatorname{Hom}_{\mathcal{M F}_{S}}\left(\iota\left(\mathcal{M}_{1}\right), \iota\left(\mathcal{M}_{2}\right)\right)=\operatorname{Hom}_{\mathrm{MF}_{S}^{e *}}\left(\mathcal{M}_{1}, \mathcal{M}_{2}\right)$ and $F \in \operatorname{Hom}_{\text {Aug }_{O}}\left(\mathcal{A}_{1}, \mathcal{A}_{2}\right)$ is such that $F \in \Psi^{*}(f)$. Then

$$
(F \otimes F)\left(\Delta_{1}\left(\bar{X}_{1}\right)\right)-\Delta_{2}\left(F\left(\bar{X}_{1}\right)\right) \in J_{A_{2} \otimes A_{2}} .
$$

Let $\bar{\alpha}=\left(\bar{\alpha}^{l o c}, \bar{\alpha}^{e t}\right)$ be the vector with the coordinates in $I_{A_{2}^{e t} \otimes A_{2}^{e t}}$ such that

$$
(F \otimes F)\left(\Delta_{1}\left(\bar{X}_{1}\right)\right)-\Delta_{2}\left(F\left(\bar{X}_{1}\right)\right) \equiv 2 \bar{\alpha} \bmod \widetilde{J}_{A_{2} \otimes A_{2}} .
$$

Note that if $F^{e t}:=\left.F\right|_{A_{1}^{e t}}$ then the congruence $\left(F^{e t} \otimes F^{e t}\right)\left(\Delta_{1}\left(\bar{X}_{1}^{e t}\right)\right) \equiv$ $\Delta_{2}\left(F^{e t}\left(\bar{X}_{1}^{e t}\right)\right) \bmod 2 A_{2}^{e t}$ implies that $\bar{\alpha}^{e t}=\overline{0}$ and $F^{e t}$ induces a morphism of etale group schemes $G_{2}^{e t} \longrightarrow G_{1}^{e t}$.

Using that $F$ is a morphism of augmented $O$-algebras we obtain that $(\bar{\alpha} C)^{(2)}+\bar{\alpha}$ has all coordinates in $\pi I_{A^{e t} \otimes A^{e t}}$.

One can verify easily that $\bar{\alpha} \bmod \pi I_{A_{2}^{e t} \otimes A_{2}^{e t}}$ has all coordinates in $Z_{\text {sym }}^{2}\left(G_{2}^{\text {et }} \otimes k\right)$ and using that for $i=1,2,2 \mathrm{id}_{G_{i}}=\Delta_{G_{i}} \circ$ mult we obtain that $\bar{\alpha} \bmod \pi I_{A_{2}^{e t} \otimes A_{2}^{e t}}$ has all coordinates in $B^{2}\left(G_{2}^{e t} \otimes k\right)$.

Let $\bar{\gamma}=\left(\gamma^{l o c}, \overline{0}\right)$ be the vector with coordinates in $I_{A_{2}^{e t}}$ such that $\bar{\gamma} \bmod \pi I_{A_{2}^{e t}}=\omega\left(\bar{\alpha} \bmod \pi I_{A_{2}^{e t} \otimes A_{2}^{e t}}\right)$. Then

$$
\Delta_{2}^{e t}(\bar{\gamma}) \equiv \bar{\gamma} \otimes 1+1 \otimes \bar{\gamma}+\bar{\alpha} \bmod \pi I_{A_{2}^{e t} \otimes A_{2}^{e t}}
$$

and $(\bar{\gamma} C)^{(2)}+\bar{\gamma}$ has all coordinates in $\pi I_{A_{2}^{e t}}$. This implies that there is an $F^{\prime} \in \operatorname{Aug}_{O}\left(\mathcal{A}_{1}, \mathcal{A}_{2}\right)$ such that $F^{\prime}\left(\bar{X}_{1}^{e t}\right)=F\left(\bar{X}_{1}^{e t}\right)$ and $F^{\prime}\left(\bar{X}^{l o c}\right) \equiv$ $F\left(\bar{X}_{1}^{l o c}\right)+2 \bar{\gamma} \bmod \widetilde{J}_{A_{2} \otimes A_{2}}$. Therefore,

$$
\left(F^{\prime} \otimes F^{\prime}\right)\left(\Delta_{1}\left(\bar{X}_{1}\right)\right) \equiv \Delta_{2}\left(F^{\prime}\left(\bar{X}_{1}\right)\right) \bmod \widetilde{J}_{A_{2} \otimes A_{2}},
$$


and $\left(F^{\prime} \otimes F^{\prime}\right) \circ \Delta_{1}=\Delta_{2} \circ F^{\prime}$. This proves the existence of $F^{\prime} \in \Psi^{*}(f)$ such that $\operatorname{Spec} F^{\prime} \in \operatorname{Hom}_{\mathrm{Gr}_{O}}\left(G_{2}, G_{1}\right)$.

Similarly, one can verify that if $F^{\prime \prime} \in \Psi^{*}(f)$ is such that $\operatorname{Spec}\left(F^{\prime \prime}\right) \in$ $\operatorname{Hom}_{\mathrm{Gr}_{O}}\left(G_{2}, G_{1}\right)$ then $F^{\prime}$ and $F^{\prime \prime}$ are equivalent in the category $\operatorname{Gr}_{O}^{*}$ with the obvious inverse statement. The proposition is proved.

1.9. Full faithfulness of $\mathcal{G}_{O}$. Suppose $\mathcal{N}=\left(N^{0}, N^{1}, \varphi_{1}\right) \in \mathrm{MF}_{S}^{e}$ and $\mathcal{G}_{O}(\mathcal{N})=\operatorname{Spec} B$.

1.9.1. Special construction of $B$. Use the following special case of the construction of the $O$-algebra $B$ from Subsection 1.4 .

Let $n_{1}^{1}, \ldots, n_{u}^{1}$ be an $S$-basis of $N^{1}$ such that there are $\tilde{s}_{1}, \ldots, \tilde{s}_{u} \in S$ and an $S$-basis $n_{1}, \ldots, n_{u}$ of $N^{0}$ such that for $1 \leqslant i \leqslant u, n_{i}^{1}=\tilde{s}_{i} n_{i}$. One can easily see that this choice of $\bar{n}^{1}=\left(n_{1}^{1}, \ldots, n_{u}^{1}\right)$ can be made in such a way that conditions $C 1$ and $C 2$ from Subsection 1.4. are satisfied. Also notice that all $\tilde{s}_{i}$ divide $t^{e}$.

Set $\bar{n}^{0}=\varphi_{1}\left(\bar{n}^{1}\right)$ and let the matrices $U \in M_{u}(S)$ and $U_{0} \in \operatorname{GL}_{u}(S)$ be such that $\bar{n}^{1}=\bar{n}^{0} U$ and $\bar{n}^{0} U_{0}=\bar{n}:=\left(n_{1}, \ldots, n_{u}\right)$. Then $U=U_{0} U_{1}$, where $U_{1}=\left(\tilde{s}_{i} \delta_{i j}\right)_{1 \leqslant i, j \leqslant u}$ is diagonal. Choose $\widetilde{\Omega}=\left(\tilde{\eta}_{i}^{\prime} \delta_{i j}\right)_{1 \leqslant i, j \leqslant u} \in$ $M_{u}(O)$ and $D \in \mathrm{GL}_{u}(O)$ such that $\kappa_{S O}\left(U_{1} \bmod t^{2 e}\right)=\widetilde{\Omega} \bmod 2$ and $\kappa_{S O}\left(U_{0} \bmod t^{2 e}\right)=D \bmod 2$. Then for $\bar{Y}=\left(Y_{1}, \ldots, Y_{u}\right)$, the $O$-algebra $B$ is the quotient of $O[\bar{Y}]:=O\left[Y_{1}, \ldots, Y_{u}\right]$ by the ideal generated by the coordinates of the vector

$$
\left((\bar{Y} D \widetilde{\Omega})^{(2)}+2 \bar{Y}\right)(D \widetilde{\Omega})^{(2)^{-1}}
$$

Then in the new coordinates $\bar{X}=\left(X_{1}, \ldots, X_{u}\right):=\bar{Y} D, B$ is the quotient of $O[\bar{X}]:=O\left[X_{1}, \ldots, X_{u}\right]$ by the ideal generated by the elements

$$
X_{i}^{2}-\eta_{i} \sum_{j} X_{j} c_{j i}, \quad 1 \leqslant i \leqslant u,
$$

Here $C=\left(c_{i j}\right)=D^{-1}$, and for all $i, \eta_{i}=-2 / \tilde{\eta}^{\prime 2}$. With this notation, the counit $e: B \longrightarrow O$ and the coaddition $\Delta: B \longrightarrow B \otimes_{O} B$ are uniquely recovered (in the category $\mathrm{Gr}_{O}^{*}$ ) from the conditions $e\left(X_{i}\right)=0$ and $\Delta\left(X_{i}\right) \equiv X_{i} \otimes 1+1 \otimes X_{i} \bmod J_{B \otimes B}$.

Remind that $\bar{X}=\left(\bar{X}^{l o c}, \bar{X}^{e t}\right)$, where for $u_{0}=\operatorname{dim}_{k} \bar{N}^{0, l o c}, \bar{X}^{l o c}=$ $\left(X_{1}, \ldots, X_{u_{0}}\right)$ and $\bar{X}^{e t}=\left(X_{u_{0}+1}, \ldots, X_{u}\right)$. Then condition $C 2$ implies:

- for $1 \leqslant i \leqslant u_{0}, X_{i}, X_{i}^{2} / \eta_{i} \in I_{B}^{\text {loc }}$;

- for $u_{0}<i \leqslant u, \eta_{i} \in O^{*}$ and $X_{i} \in I_{B^{e t}}$.

Therefore, the matrix $C=\left(c_{i j}\right)$ has the following block structure $C=\left(\begin{array}{cc}C_{0} & 0 \\ C^{\prime} & C^{e t}\end{array}\right)$, where $C_{0} \in \mathrm{GL}_{u_{0}}(O), C^{e t} \in \mathrm{GL}_{u^{e t}}(O)$ with $u^{e t}:=$ $u-u_{0}, C^{\prime} \equiv 0 \bmod \pi$. In particular,

(C) if $C=\left(c_{i j}\right)$ and $D=C^{-1}=\left(d_{i j}\right)$ then $c_{i j} \equiv d_{i j} \equiv 0 \bmod \pi$ if either $i \leqslant u_{0}<j$ or $j \leqslant u_{0}<i$. 
Let $\delta^{+} X_{i}=j_{i} \in I_{B \otimes B}, 1 \leqslant i \leqslant u$. The coordinates of the vector $\bar{\jmath}=\left(\bar{\jmath}^{o c}, \bar{\jmath}^{e t}\right)$, where $\bar{\jmath}^{l o c}=\left(j_{1}, \ldots, j_{u_{0}}\right)$ and $\bar{\jmath}^{e t}=\left(j_{u_{0}+1}, \ldots, j_{u}\right)$, appear as the solutions in $I_{B \otimes B}$ of the system of equations

$$
\sum_{s} j_{s} c_{s i}=-\tilde{\eta}_{i} X_{i} \otimes X_{i}-\left(\tilde{\eta}_{i} X_{1} \otimes 1+1 \otimes \tilde{\eta}_{i} X_{i}\right) j_{i}-\tilde{\eta}_{i} j_{i}^{2} / 2
$$

where $1 \leqslant i \leqslant u$ and $\tilde{\eta}_{i}=-2 / \eta_{i}=\tilde{\eta}^{2}$.

The coordinates of $\bar{j}^{\text {et }}$ are determined by these equations uniquely and belong to the ideal $J_{B}$. The coordinates of $\bar{\jmath}^{l o c}$ are unique under the assumption that $j_{1}, \ldots, j_{u_{0}} \in \widetilde{J}_{B \otimes B} \subset J_{B \otimes B}$.

Remark. a) One can easily verify that the above system of equations when considered modulo any $D P$-ideal $\widetilde{I}$ of $B$ such that $\widetilde{I} \subset \widetilde{J}_{B \otimes B}$ has a unique solution $\bar{\jmath} \bmod \widetilde{I}$ under the assumption that for $1 \leqslant i \leqslant u_{0}$, all $j_{i} \in \widetilde{J}_{B \otimes B}$.

b) The above description of the coalgebra $B$ is related to a very special choice of $S$-bases in $N^{0}$ and $N^{1}$. This choice is sufficient for the formal construction of the algebras $\mathcal{A}(\mathcal{M})$ in Subsection 1.3. But when proving the full faithfulness of $\mathcal{G}_{O}$ below in Section 3 we need a choice of appropriate bases which is compatible with extensions in $\mathrm{MF}_{S}^{e}$. Such choice is possible under more general assumptions from Subsection 1.3 .

1.9.2. Recovering $\mathcal{N}$. In the above construction of the $O$-algebra $B$, any $a \in I_{B}$ can be uniquely written as

$$
a=\sum_{\underline{i}} o_{\underline{i}} X^{\underline{i}}=\sum_{\underline{i}} o_{\underline{i}} X_{1}^{i_{1}} \ldots X_{u}^{i_{u}}
$$

where (by our general agreement from Introduction) $\underline{i}=\left(i_{1}, \ldots, i_{u}\right)$ is a non-zero vector with the coordinates $i_{1}, \ldots, i_{u} \in\{0,1\}$, and all coefficients $o_{\underline{i}}=o_{\underline{i}}(a)$ belong to $O$. Similarly, any $a \in I_{B \otimes B}$ can be uniquely written as an $O$-linear combination of the elements $X^{\underline{i}_{1}} \otimes 1$, $1 \otimes X \underline{i}_{2}$ and $X^{\underline{i}_{1}} \otimes X \underline{\underline{i}}_{2}$

Consider the following property of ideals $I \subset I_{B}$ in $B$ (or with the obvious changes in $B \otimes B, \bar{B}, \bar{B} \otimes \bar{B}$, etc.).

$$
\sum_{\underline{i}} o_{\underline{i}} X^{\underline{i}} \in I \Leftrightarrow \underline{i}, \quad o_{\underline{i}} X^{\underline{i}} \in I
$$

Remark. Suppose $I_{1}$ and $I_{2}$ satisfy property (1.5). Then

a) $I_{1}+I_{2}$ satisfies property (1.5);

b) for any monomial $o_{\underline{i}} X^{\underline{i}}, o_{\underline{i}} X^{\underline{i}} \in I_{1}+I_{2}$ iff either $o_{\underline{i}} X^{\underline{i}} \in I_{1}$ or $o_{\underline{i}} X^{\underline{i}} \in I_{2}$.

Proposition 1.13. The ideals $J_{B}$ and $\widetilde{J}_{B}$ satisfy property (1.5). 
Proof. Any element of $J_{B}$ is a sum of "elementary" elements of the form $o_{\underline{i}_{1}} X^{\underline{i}_{1}} O_{\underline{i}_{2}} X^{\underline{i}_{2}}$ and $\pi^{e} O_{\underline{i}_{1}} X^{\underline{i}_{1}}$, where $o_{\underline{i}_{1}} X^{\underline{i}_{1}}, o_{\underline{i}_{2}} X^{\underline{i}_{2}} \in I_{B}(2)$. It will be sufficient to verify property (1.5) for such elementary elements.

If $a=\pi^{e} O_{\underline{i}_{1}} X^{\underline{i}_{1}}$ there is nothing to prove.

Suppose $a=o_{\underline{i}_{1}} X^{\underline{i}_{1}} O_{\underline{i}_{2}} X \underline{\underline{i}}_{2}$ and $\underline{i}_{1}=\left(i_{11}, \ldots, i_{u 1}\right), \underline{i}_{2}=\left(i_{12}, \ldots, i_{u 2}\right)$. Use induction on the number $\nu=\nu(a)$ of $1 \leqslant j \leqslant u$ such that $i_{j 1}=$ $i_{j 2}=1$.

If $\nu(a)=0$ then $\underline{i}_{1}+\underline{i}_{2}=\underline{i}$ and there is nothing to prove. If $\nu(a) \geqslant 1$ and, say, $i_{j 1}=i_{j 2}=1$ use the identity $X_{j}^{2}=\eta_{j}^{\prime} \cdot \eta_{j}^{\prime} \sum_{s} X_{s} c_{s j}$ to rewrite $a$ as a sum of elements with smaller $\nu$ 's and, perhaps, elements of the form $\pi^{e} I_{B}(2)$.

The case of the ideal $\widetilde{J}_{B}$ can be considered similarly.

Proposition 1.14. If $\iota_{\mathcal{N}}(\mathcal{N})=\left(\tilde{N}^{0}, \tilde{N}^{1}, \varphi_{1}\right)$ then

$$
\widetilde{N}^{0}=\left\{a \bmod J_{B} \mid a \in I_{B}, \delta^{+}(a) \in J_{B \otimes B}\right\}
$$

Proof. Suppose $a=\sum_{\underline{i}} o_{\underline{i}} X^{\underline{i}} \in I_{B}$ and $\delta^{+} a \in J_{B \otimes B}$. Note that

$$
\delta^{+}(a) \equiv \sum_{\underline{i}_{1}+\underline{i}_{2}=\underline{i}} o_{\underline{i}} X^{\underline{i}_{1}} \otimes X^{\underline{i}_{2}} \bmod J_{B \otimes B}
$$

Then Proposition 1.13 implies that all $o_{\underline{i}} X^{\underline{i}_{1}} \otimes X^{\underline{i}_{2}} \in J_{B \otimes B}$ and, therefore, all $o_{\underline{i}} X^{\underline{i}_{1}} \cdot X^{\underline{i}_{2}} \in J_{B}$. This means that all non-linear terms amongst $o_{\underline{i}} X \underline{i}$ (i.e. the terms with $r(\underline{i})=i_{1}+\cdots+i_{u} \geqslant 2$ ) belong to $J_{B}$.

Using that the ideals $J_{B}$ and $J_{B \otimes B}$ depend functorially on the group scheme $\mathcal{G}_{O}(\mathcal{N})$ (i.e. do not depend on a choice of the special construction in Subsection 1.9) we obtain the following property.

Corollary 1.15. The functor $\mathcal{G}_{O}$ is fully faithful.

Proof. Suppose $G_{1}=\mathcal{G}_{O}\left(\mathcal{M}_{1}\right), G_{2}=\mathcal{G}_{O}\left(\mathcal{M}_{2}\right), \mathcal{M}_{1}, \mathcal{M}_{2} \in \mathrm{MF}_{S}^{e}, g \in$ $\operatorname{Hom}_{\mathrm{Gr}_{\mathrm{O}}}\left(G_{1}, G_{2}\right)$ and $A(g): A_{2} \longrightarrow A_{1}$ is the corresponding morphism of $O$-algebras. Then $\iota(A(g)) \in \operatorname{Hom}_{\mathcal{M F}_{S}}\left(\iota\left(A_{2}\right), \iota\left(A_{1}\right)\right)$ maps $\iota_{\mathcal{M}_{2}}\left(\mathcal{M}_{2}\right)$ to $\iota_{\mathcal{M}_{1}}\left(\mathcal{M}_{1}\right)$ (use Proposition 1.14) and by Proposition 1.1 can be lifted uniquely $F \in \operatorname{Hom}_{\mathrm{MF}_{S}^{e}}\left(\mathcal{M}_{2}, \mathcal{M}_{1}\right)$. Clearly, $\mathcal{G}_{O}(F)=g$.

In Subsection 3 we need the following version of Proposition 1.14 .

Let $\bar{B}=B \otimes_{O} \bar{O}$. Denote by $\overline{\mathcal{J}}$ the ideal in $\bar{B}$ generated by $\tilde{\eta}_{i} X_{i} \otimes X_{i}$, $1 \leqslant i \leqslant u_{0}$, and all elements of $2 I_{\bar{B} \otimes \bar{B}}^{l o c}$. One can easily prove (use relation (1.3)) that all $j_{1}, \ldots, j_{u_{0}}$ belong to $\overline{\mathcal{J}}$ and $\pi j_{u_{0}+1}, \ldots, \pi j_{u} \in$ $2 \pi I_{B \otimes B} \subset \overline{\mathcal{J}}$.

Proposition 1.16. The ideal $\overline{\mathcal{J}}$ consists of all $\bar{O}$-linear combinations of monomials in $\bar{B} \otimes \bar{B}$ which either belong to $2 I_{\bar{B} \otimes \bar{B}}^{\text {loc }}$ or are divisible by one of $\tilde{\eta}_{i} X_{i} \otimes X_{i}$, where $1 \leqslant i \leqslant u_{0}$. 
Proof. Suppose an element $a \in \overline{\mathcal{J}}$ is an $\bar{O}$-linear combination of the form

$$
a=\sum_{\underline{i}} o_{\underline{i}}^{\prime}\left(X^{\underline{i}} \otimes 1\right)+\sum_{\underline{i}} o_{\underline{i}}^{\prime \prime}\left(1 \otimes X^{\underline{i}}\right)+\sum_{\underline{i}_{1}, \underline{i}_{2}} o_{\underline{i}_{1} \underline{i}_{2}} X^{\underline{i}_{1}} \otimes X^{\underline{i}_{2}}
$$

Let $M(i, b)=\tilde{\eta}_{i}\left(X_{i} \otimes X_{i}\right) b$, where $1 \leqslant i \leqslant u_{0}$ and $b$ is a monomial from $\bar{B} \otimes \bar{B}$.

Clearly, any $M(i, b)$ can't contribute to the coefficients of the first two sums in (1.6). Therefore, their summands belong to $2 I_{\bar{B} \otimes \bar{B}}^{l o c}$.

Suppose $o_{\underline{i}_{1} \underline{i}_{2}} X^{\underline{i}_{1}} \otimes X \underline{\underline{i}}_{2} \notin 2 I_{\bar{B} \otimes \bar{B}}^{l o c}$ and satisfies the following condition (The proposition is proved if there are no such monomials.):

if $X^{\underline{i}_{1}} \otimes X^{\underline{i}_{2}}$ is divisible by $X_{i} \otimes X_{i}, 1 \leqslant i \leqslant u_{0}$, then $o_{\underline{\underline{i}}_{1} \underline{\underline{i}}_{2}}$ is not divisible by $\tilde{\eta}_{i}$.

Then there is $M\left(i_{0}, b\right)$ with $1 \leqslant i_{0} \leqslant u_{0}$, which contributes to the coefficient for $X^{\underline{i_{1}}} \otimes X^{\underline{i}_{2}}$ and this contribution divides $o_{\underline{i}_{1} \underline{i}_{2}}$.

If $b$ is not divisible by either $X_{i_{0}} \otimes 1$ or $1 \otimes X_{i_{0}}$ then $M\left(i_{0}, b\right)=$ $o X^{\underline{i}_{1}} \otimes X^{\underline{i}_{2}}$ and $o \in \bar{O}$ is divisible by $\tilde{\eta}_{i_{0}}$. The contradiction. But otherwise, $M\left(i_{0}, b\right) \in 2 I_{B}^{\text {loc }}$ because $\tilde{\eta}_{i_{0}} X_{i_{0}}^{2} \in 2 I_{B}^{\text {loc }}$. The proposition is proved.

Corollary 1.17. The ideal $\overline{\mathcal{J}}$ satisfies condition (1.5).

\section{FunCTOR $\mathcal{G}_{O_{0}}^{O}$}

In this section we prove that any $G \in \mathrm{Gr}_{O}$ from the image of $\mathcal{G}_{O}$ has a canonical descent to $O_{0}, G_{0} \in \mathrm{Gr}_{O_{0}}$. Therefore, the fully faithful functor $\mathcal{G}_{O}$ appears as the composition of the fully faithful functor $\mathcal{G}_{O_{0}}^{O}: \mathrm{MF}_{S}^{e *} \longrightarrow \mathrm{Gr}_{O_{0}}^{*}$ and the extension of scalars $\mathrm{Gr}_{O_{0}}^{*} \longrightarrow \mathrm{Gr}_{O}^{*}$.

\subsection{Uniqueness of descent to $O_{0}$.}

Proposition 2.1. a) Suppose $G=\operatorname{Spec} A \in \operatorname{Im} \mathcal{G}_{O}, e: A \longrightarrow O$ is the counit and $I_{A}=$ Ker $e$. If there is an $\left(A_{0}, I_{A_{0}}\right) \in \operatorname{Aug}_{O_{0}}$ such that $\left(A_{0}, I_{A_{0}}\right) \otimes_{O_{0}} O=\left(A, I_{A}\right)$ then $G_{0}=\operatorname{Spec} A_{0}$ has a natural structure of object of the category $\mathrm{Gr}_{O_{0}}$ such that $G_{0} \otimes_{O_{0}} O=G$.

b) Suppose $G_{0}, H_{0} \in \mathrm{Gr}_{O_{0}}$ and $G=G_{0} \otimes_{O_{0}} O$ and $H=H_{0} \otimes_{O_{0}} O$ are in the image of $\mathcal{G}_{O}$. Then the natural map $f \mapsto f \otimes_{O_{0}} O$ induces identification $\operatorname{Hom}_{\mathrm{Gr}_{0}}\left(G_{0}, H_{0}\right)=\operatorname{Hom}_{\mathrm{Gr}_{O}}(G, H)$.

Proof. It will be sufficient to prove that $\Delta\left(I_{A_{0}}\right) \subset I_{A_{0} \otimes A_{0}}$, where $\Delta$ is the coaddition on $A$.

Let $\operatorname{Gal}\left(K / K_{0}\right)=\{\mathrm{id}, \tau\}$ and $\Delta^{(\tau)}=(\tau \otimes \tau) \circ \Delta \circ \tau$ is the conjugate to $\Delta$. In other words, if $b_{1}, \ldots, b_{u}$ is an $O_{0}$-basis of $I_{A_{0}}$ and for $1 \leq i \leq u$, $\Delta\left(b_{i}\right)=b_{i} \otimes 1+1 \otimes b_{i}+\sum_{k, l} \alpha_{k l} b_{k} \otimes b_{l}$ with all $\alpha_{k l} \in O$, then $\Delta^{(\tau)}\left(b_{i}\right)=$ $b_{i} \otimes 1+1 \otimes b_{i}+\sum_{k, l} \tau\left(\alpha_{k l}\right) b_{k} \otimes b_{l}$. Using that all $\tau\left(\alpha_{k l}\right) \equiv \alpha_{k l} \bmod 2 \pi O$, we conclude that for any $a \in I_{A}, \Delta^{(\tau)}(a) \equiv \Delta(a) \bmod \widetilde{J}_{A \otimes A}$. Therefore, 
by results of Subsection [1.7, $\Delta=\Delta^{(\tau)}$ and all $\alpha_{k l} \in O_{0}$. Part a) is proved.

Part b) follows by similar arguments.

\subsection{Existence of descent to $O_{0}$.}

Proposition 2.2. Suppose $G=\operatorname{Spec} A \in \operatorname{Im} \mathcal{G}_{O}$ and $I_{A}=\operatorname{Ker} e$, where $e: A \longrightarrow O$ is the counit. Then there is an $\left(A_{0}, I_{A_{0}}\right) \in \operatorname{Aug}_{O_{0}}$ such that $I_{A}=I_{A_{0}} \otimes_{O_{0}} O$.

Proof. Use induction on the order $|G|$ of $G$.

2.2.1. The case $|G|=2$. Here $A=O[X]$, where $X^{2}=\eta c X$ with $\eta \in O_{0}, \eta \mid 2$ and $c \in O^{*}$. Clearly, we can take $A_{0}=O_{0}\left[c^{-1} X\right]$.

2.2.2. Tame descent. Suppose $K_{0}^{\prime}$ is a tamely ramified extension of $K_{0}$ of degree $e_{0}$. Let $\pi_{0}^{\prime}$ be a uniformising element of $K_{0}^{\prime}$ such that $\pi_{0}^{\prime e_{0}}=\pi_{0}$. Let $K^{\prime}=K_{0}^{\prime}\left(\pi^{\prime}\right)$, where $\pi^{\prime 2}=\pi_{0}^{\prime}$. We can assume that $\pi^{\prime e_{0}}=\pi$. The field extensions $K_{0}^{\prime} / K_{0}$ and $K^{\prime} / K$ are Galois, their Galois groups are cyclic of order $e_{0}$ and can be naturally identified. Denote by $O_{0}^{\prime}$ and $O^{\prime}$ the valuation rings of $K_{0}^{\prime}$ and, resp., $K^{\prime}$.

Lemma 2.3. Let $G \in \mathrm{Gr}_{O}$ and $G^{\prime}=G \otimes_{O} O^{\prime} \in \mathrm{Gr}_{O^{\prime}}$. Then:

a) $G$ is in the image of $\mathcal{G}_{O}$ if and only if $G^{\prime}$ is in the image of $\mathcal{G}_{O^{\prime}}$;

b) $G$ admits a descent to $O_{0}$ if and only if $G^{\prime}$ admits a descent to $O_{0}^{\prime}$.

Proof. The proof is based on an application of the criterion of tamely ramified descent. In the case of $O^{\prime}$-algebras this criterion can be stated as follows:

- Suppose $A^{\prime}$ is a flat $O^{\prime}$-algebra and $\tau$ is a generator of $\operatorname{Gal}\left(K^{\prime} / K\right)$. Then the existence of a flat $O$-algebra $A$ such that $A^{\prime}=A \otimes_{O} O^{\prime}$ is equivalent to the existence of a $\tau$-linear automorphism $f$ of $A^{\prime}$ such that $f^{e_{0}}=\operatorname{id}_{A^{\prime}}$ and $f \otimes_{O} k=\operatorname{id}_{A^{\prime} \otimes k}$.

Then one can state a similar criterion for objects of $\mathrm{MF}_{S^{\prime}}^{e_{0} e}$ where $S^{\prime}$ is a tamely ramified extension of $S$ of degree $e_{0}$ and deduce part a) from the fact that tame descent data for $G^{\prime}=\mathcal{G}_{O^{\prime}}\left(\mathcal{M}^{\prime}\right)$ induce tame descent data for $\mathcal{M}^{\prime}$. Similarly, part b) can be proved from the fact that tamely ramified descent data for $G^{\prime}$ induce tamely ramified descent data for $G_{0}^{\prime}$ (use the uniqueness of $G_{0}^{\prime}$ given by Proposition 2.1). Cf. for more detailed explanation in [4], Proposition 4.3.

2.2.3. Lubin-Tate formal group law. Consider the formal Lubin-Tate group law with the logarithm

$$
l_{L T}(X)=X+X^{2} / 2+\cdots+X^{2^{n}} / 2^{n}+\cdots \in \mathbb{Q}_{2}[[X]]
$$


This means that for any 2-adic ring $R$ its topological nilradical $\mathrm{m}(R)$ is provided with the structure of abelian group via the operation $[f]+[g]=$ $[P(f, g)]$, where $f, g \in \mathrm{m}(R)$ and for indeterminants $X, Y$,

$$
P(X, Y)=l_{L T}^{-1}\left(l_{L T}(X)+l_{L T}(Y)\right) \in \mathbb{Z}_{2}[[X, Y]] .
$$

We have the following simple properties:

- $[f]+[g]=\left[P_{0}(f, g)\right]+\cdots+\left[P_{n}(f, g)\right]+\ldots$, where all $P_{n} \in \mathbb{Z}_{2}[X, Y]$ and $\operatorname{deg} P_{n}=2^{n}$. In particular, $P_{0}=X+Y, P_{1}=-X Y, P_{2}=$ $-X Y(X+Y)^{2}$

- $-[f]=[-f]+\left[-f^{2}\right]+\cdots+\left[-f^{2^{n}}\right]+\ldots$

- $[2](f)=[f]+[f] \equiv[2 f]+\left[f^{2}\right]+\left[-2 f^{2}\right]+\cdots+\left[-2 f^{2^{n}}\right]+\ldots \bmod 4 \mathrm{~m}(R)$.

2.2.4. The case $|G|>2$. By replacing $O$ by its suitable tamely ramified extension we can assume that $G=\mathcal{G}_{O}(\mathcal{M})$, where $\mathcal{M}=\left(M^{0}, M^{1}, \varphi_{1}\right) \in$ $\mathrm{MF}_{S}^{e}$ is such that for $u \geqslant 1$ :

- there is an $S$-basis $m^{1}, n_{1}^{1}, \ldots, n_{u}^{1}$ of $M^{1}$ and an $S$-basis $m, n_{1}, \ldots, n_{u}$ of $M^{0}$ such that $\varphi_{1}\left(m^{1}\right)=m$ and for $1 \leqslant i \leqslant u$, there are $\tilde{s}_{i} \in S, \tilde{s}_{i} \mid t^{e}$ such that $n_{i}^{1}=\tilde{s}_{i} n_{i}, \varphi_{1}\left(n_{1}^{1}\right)=\sum_{j} n_{j} u_{j i}$, where $\left(u_{i j}\right) \in \operatorname{GL}_{u}(S)$;

- there is an $\tilde{s} \in S, \tilde{s} \mid t^{e}$ such that $m^{1}=\tilde{s} m+\sum_{i} \alpha_{i} n_{i}$, where for $1 \leqslant i \leqslant u$, the coefficients $\alpha_{i} \in S$ and $t^{e} \tilde{s}^{-1} \alpha_{i} \equiv 0 \bmod \tilde{s}_{i}$.

The above conditions simply mean that $\mathcal{N}=\left(N^{0}, N^{1}, \varphi_{1}\right) \in \mathrm{MF}_{S}^{e}$, where $N^{0}=\sum_{i} S n_{i}, N^{1}=\sum_{i} S n_{i}^{1}$ and there is a short exact sequence in $\mathrm{MF}_{S}^{e}$

$$
0 \longrightarrow \mathcal{N} \longrightarrow \mathcal{M} \longrightarrow \mathcal{M}_{\tilde{s}} \longrightarrow 0
$$

where $\mathcal{M}_{\tilde{s}}=\left(S \tilde{m}, S \tilde{m}^{1}, \varphi_{1}\right)$ with $\tilde{m}^{1}=\tilde{s} \tilde{m}$ and $\varphi_{1}\left(\tilde{m}^{1}\right)=\tilde{m}$. We shall always assume that the above data for the structure of $\mathcal{N} \in \mathrm{MF}_{S}^{e}$ satisfy assumptions $C 1$ and $C 2$ from Subsection 1.4.

Note that in the above description of $\mathcal{M} \in \operatorname{Ext}_{\mathrm{MF}_{S}^{e}}\left(\mathcal{M}_{\tilde{s}}, \mathcal{N}\right)$ we can replace $m$ by $m^{\prime}=m+v$ and $m^{1}$ by $m^{1 \prime}=m^{1}+v^{1}$, where $v \in N^{0}$, $v^{1} \in N^{1}$ and $\varphi_{1}\left(v^{1}\right)=v$. Then $m^{1 \prime}=\tilde{s} m^{\prime}+\sum_{i} \alpha_{i}^{\prime} n_{i}$, where

$$
\sum_{i} \alpha_{i}^{\prime} n_{i}=\sum_{i} \alpha_{i} n_{i}+v^{1}-\tilde{s} \varphi_{1}\left(v^{1}\right)
$$

In particular, if $\tilde{s} \in S^{*}$ we can always assume that

$$
\sum_{i} \alpha_{i} n_{i} \in N^{1}+t N^{0}
$$

In terms of the corresponding $O$-algebras we have:

- $A=A(G)$ contains the $O$-algebra $B=O\left[X_{1}, \ldots, X_{u}\right]$, where $X_{i}^{2}=$ $\eta_{i} \sum_{j} X_{j} c_{j i}$ with $\eta_{i}=-2 / \tilde{\eta}_{i}^{\prime 2} \in O_{0}$ and $\tilde{\eta}_{i}^{\prime} \bmod 2=\kappa_{S O}\left(\tilde{s}_{i} \bmod t^{2 e}\right)$, $1 \leqslant i \leqslant u$, and $C=\left(c_{i j}\right) \in \mathrm{GL}_{u}(O)$; 
- $A=B[Y]$, where $\left(\tilde{\eta}^{\prime} Y+\sum_{i} r_{i} X_{i}\right)^{2}+2 Y=0$ or, equivalently,

$$
\left(Y+\tilde{\eta}^{\prime-1} \sum_{i} r_{i} X_{i}\right)^{2}-\eta Y=0
$$

with $\tilde{\eta}^{\prime} \bmod 2=\kappa_{S O}\left(\tilde{s} \bmod t^{2 e}\right), r_{i} \bmod 2=\kappa_{S O}\left(\alpha_{i} \bmod t^{2 e}\right)$ and

$$
\eta r_{i}^{2} \equiv 0 \bmod \tilde{\eta}_{i}\left(\text { or, equivalently, } \eta_{i} r_{i}^{2} \equiv 0 \bmod \tilde{\eta}\right)
$$

for all $1 \leqslant i \leqslant u$ (this follows from the above congruences $t^{e} \tilde{s}^{-1} \alpha_{i} \equiv$ $0 \bmod \tilde{s}_{i}$ ). Recall that as usually $\eta \tilde{\eta}=\eta_{i} \tilde{\eta}_{i}=-2$ for all $i$.

Let $h=\sum_{i} r_{i} X_{i} \in I_{B}$. Then (2.3) implies that $h^{2} \in \tilde{\eta} I_{B}$. In particular, if $\tilde{\eta} \notin O_{0}^{*}$ then $h \in I_{B}^{\text {loc }}$. If $\tilde{\eta} \in O_{0}^{*}$ then (2.1) implies that again $h \in I_{B}^{\text {loc }}$. Therefore, $\tilde{\eta}^{\prime} Y \in I_{A}^{\text {loc }}$.

By inductive assumption, there is an augmented flat $O_{0}$-algebra $\left(B_{0}, I_{B_{0}}\right)$ such that $I_{B}=I_{B_{0}} \otimes_{O_{0}} O$. Therefore, $h=b_{0}+\pi b_{1}$, where $b_{0} \in I_{B_{0}}^{l o c}$ and $b_{1} \in I_{B_{0}}$.

From now on we use the Lubin-Tate group law. Clearly, there is $Y^{\prime} \in I_{A}$ such that $\left[\tilde{\eta} Y^{\prime}\right]=\left[\tilde{\eta} Y+\tilde{\eta}^{\prime} h\right]-\left[\tilde{\eta}^{\prime} b_{0}\right]-\left[\tilde{\eta}^{\prime} \pi b_{1}\right]$. If $\tilde{\eta} \notin O_{0}^{*}$ then $Y^{\prime} \equiv Y \bmod \pi I_{A}$ and if $\tilde{\eta} \in O_{0}^{*}$ then $Y^{\prime} \equiv Y \bmod \left(Y I_{A}^{l o c}+\pi I_{B}^{l o c}\right)$. Therefore, $A=B\left[Y^{\prime}\right]$.

The equation for $Y^{\prime}$ can be found as follows. From (2.2) we obtain that $\left(\tilde{\eta} Y+\tilde{\eta}^{\prime} h\right)^{2}=-2 \tilde{\eta} Y$. Then using the properties of the Lubin-Tate group law from Subsection 2.2 .3 we obtain:

$$
\begin{gathered}
{[2]\left(\tilde{\eta} Y^{\prime}\right)=\left[2\left(\tilde{\eta} Y+\tilde{\eta}^{\prime} h\right)\right]+[-2 \tilde{\eta} Y]-\left([2]\left(\tilde{\eta}^{\prime} b_{0}\right)+[2]\left(\tilde{\eta}^{\prime} \pi b_{1}\right)\right) \equiv} \\
-\left[\tilde{\eta} b_{0}^{2}\right]-\left[\tilde{\eta} \pi_{0} b_{1}^{2}\right]-\sum_{n \geqslant 0}\left[-2\left(\tilde{\eta} b_{0}^{2}\right)^{2^{n}}\right]-\sum_{n \geqslant 0}\left[-2\left(\tilde{\eta} \pi_{0} b_{1}^{2}\right)^{2^{n}}\right] \bmod 4\left(\tilde{\eta} I_{A}\right)^{l o c}
\end{gathered}
$$

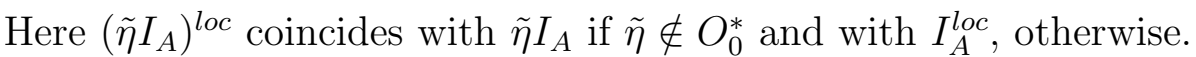

Notice that $h^{2} \in \tilde{\eta} I_{B}$ and, therefore, the right hand side of the above congruence equals $\tilde{\eta}^{2} b^{*} \in\left(\tilde{\eta}^{2} I_{B_{0}}\right)^{l o c}$. Using that $[2]\left(2\left(\tilde{\eta} I_{A}\right)^{l o c}\right)=$ $4\left(\tilde{\eta} I_{A}\right)^{l o c}$ we can replace $Y^{\prime}$ by $Y_{1}=Y^{\prime}-2 a$ with a suitable $a \in I_{A}$ such that $[2]\left(\tilde{\eta} Y_{1}\right)=\tilde{\eta}^{2} b^{*}$.

Finally, if $1+\tilde{\eta} Y_{2}=\exp \left(l_{L T}\left(\tilde{\eta} Y_{1}\right)\right)$ then we still have $A=B\left[Y_{2}\right]$ and $\left(1+\tilde{\eta} Y_{2}\right)^{2}=\exp \left(l_{L T}\left(\tilde{\eta}^{2} b^{*}\right)\right)$ implies that $Y_{2}^{2}-\eta Y_{2}=b_{1}^{*}$ with $b_{1}^{*} \in B_{0}$. So, for $A_{0}=B_{0}\left[Y_{2}\right], A_{0} \otimes_{O_{0}} O=A$.

\section{Surjectivity of $\mathcal{G}_{O_{0}}^{O}$}

In order to establish that $\mathcal{G}_{O_{0}}^{O}$ is antiequivalence of the categories $\mathrm{MF}_{S}^{e *}$ and $\mathrm{Gr}_{O_{0}}^{*}$ it remains only to prove the following result.

Theorem 3.1. If $G_{0} \in \mathrm{Gr}_{O_{0}}$ then there is $\mathcal{M} \in \mathrm{MF}_{S}^{e}$ such that $\mathcal{G}_{O}(\mathcal{M}) \simeq G_{0} \otimes_{O_{0}} O$. 
The proof will be given in Subsections 3.1-3.11. It uses induction on the order $\left|G_{0}\right|$ of $G_{0}$.

3.1. The case $\left|G_{0}\right|=2$. If $\left|G_{0}\right|=2$ then there is $\eta \in O_{0}, \eta \mid 2$, such that $G_{0}=\mu_{\eta}$, where $\mu_{\eta}=\operatorname{Spec} O_{0}[X], X^{2}=\eta X, e(X)=0$ and $\Delta(X)=X \otimes 1+1 \otimes X+\tilde{\eta} X \otimes X$ with $\eta \tilde{\eta}=-2$. We can assume that $\tilde{\eta}=\pi_{0}^{r}, 0 \leqslant r \leqslant e$ (because $\mu_{\eta} \simeq \mu_{\eta^{\prime}}$ iff $\eta^{-1} \eta^{\prime} \in O_{0}^{*}$ ). Then for $\mathcal{M}=\left(S m, S t^{r} m, \varphi_{1}\right) \in \mathrm{MF}_{S}^{e}$ with $\varphi_{1}\left(t^{r} m\right)=m$, one has $\mathcal{G}_{O}(\mathcal{M})=$ $\mu_{\eta} \otimes_{O_{0}} O$.

3.2. Basic strategy I. When studying the case $\left|G_{0}\right|>2$ we can replace $O_{0}$ by its tamely ramified extension, cf. Subsection 2.2.2. In particular, we can assume that in $\mathrm{Gr}_{O_{0}}$ there is a short exact sequence

$$
0 \longrightarrow \mu_{\eta} \longrightarrow G_{0} \longrightarrow H_{0} \longrightarrow 0,
$$

where $H_{0}=\operatorname{Spec} B_{0}$, and $H=H_{0} \otimes_{O_{0}} O=\mathcal{G}_{O}(\mathcal{N})$ with $\mathcal{N} \in \mathrm{MF}_{S}^{e}$.

Use the description of $B=B_{0} \otimes_{O_{0}} O$ from Subsection 1.9.1.

Namely, $B=O\left[X_{1}, \ldots, X_{u}\right]$ with the relations $X_{i}^{2}=\eta_{i} \sum_{j} X_{j} c_{j i}$, where all $\eta_{i} \in O_{0}, \eta_{i} \mid 2, C=\left(c_{i j}\right) \in \mathrm{GL}_{u}(O), e\left(X_{i}\right)=0$ and $j_{i}=$ $\delta^{+}\left(X_{i}\right)=\Delta\left(X_{i}\right)-X_{i} \otimes 1-1 \otimes X_{i} \in J_{B \otimes B}$. In addition, if $\bar{X}^{l o c}=$ $\left(X_{1}, \ldots, X_{u_{0}}\right)$ then $j_{1}, \ldots, j_{u_{0}} \in \widetilde{J}_{B \otimes B}$. For $1 \leqslant i \leqslant u$, the elements $\eta_{i}$ are defined up to units in $O_{0}$ and we can assume that $\tilde{\eta}_{i}=\tilde{\eta}_{i}^{\prime 2}=$ $-2 / \eta_{i}$ with $\tilde{\eta}_{i}^{\prime} \in O$. Our strategy is to use the explicit construction of $H=H_{0} \otimes_{O_{0}} O$ from Subsection 1.9.1 to describe $G=G_{0} \otimes_{O_{0}} O$ as an element of the group $\operatorname{Ext}_{\mathrm{Gr}_{O}}\left(H, \mu_{\eta} \otimes_{O_{0}} O\right)$. If $O^{\prime}=O\left[\pi^{\prime}\right]$ with $\pi^{\prime 2}=\pi$, we shall prove then that $G^{\prime}=G \otimes_{O} O^{\prime}$ appears in the form $\mathcal{G}_{O^{\prime}}\left(\mathcal{M}^{\prime}\right)$, where $\mathcal{M}^{\prime} \in \mathrm{MF}_{S^{\prime}}^{2 e}$ with $S^{\prime}=S\left[t^{\prime}\right], t^{\prime 2}=t$.

Finally, we shall prove that the fact that $G^{\prime}$ admits a descent $G_{0}$ to $O_{0}$ implies that $\mathcal{M}^{\prime}$ admits a descent to $S$, i.e. $\mathcal{M}^{\prime}=\mathcal{M} \otimes_{S} S^{\prime}$ with $\mathcal{M} \in \mathrm{MF}_{S}^{e}$ and, therefore, $G=\mathcal{G}_{O}(\mathcal{M})$.

3.3. The group $\operatorname{Ext}_{\operatorname{Gr}_{O_{0}}}\left(H_{0}, \mu_{\eta}\right)$. Let $B^{\prime}=B \otimes_{O} O^{\prime}, I_{B^{\prime}}=I_{B} \otimes_{O} O^{\prime}$. Let $\bar{O}$ be the valution ring of an algebraic closure of $K^{\prime}=K\left(\pi^{\prime}\right)$, $\bar{B}=B^{\prime} \otimes_{O^{\prime}} \bar{O}$ and $I_{\bar{B}}=I_{B^{\prime}} \otimes_{O^{\prime}} \bar{O}$.

Introduce the (multiplicative) group $\mathcal{H}=\mathcal{H}\left(H_{0}, \mu_{\eta}\right)$ of all elements $f \in\left(1+\tilde{\eta} I_{\bar{B}}\right)^{\times}$such that $f^{2} \in 1+\tilde{\eta}^{2} I_{B_{0}}$ and $\delta^{\times}(f):=\Delta(f)(f \otimes f)^{-1} \in$ $1+\tilde{\eta} I_{B_{0} \otimes B_{0}}$.

Then there is a group epimorphism $\Theta: \mathcal{H} \longrightarrow \operatorname{Ext}_{\operatorname{Gr}_{O_{0}}}\left(H_{0}, \mu_{\eta}\right)$ attaching to $f \in \mathcal{H}$, the group scheme $\Theta(f)=\operatorname{Spec} A_{0} \in \operatorname{Gr}_{O_{0}}$ such that:

- $A_{0}=B_{0}[X]$ where $(1+\tilde{\eta} X)^{2}=f^{2}$;

- $e(X)=0$ and $\Delta(1+\tilde{\eta} X)=(1+\tilde{\eta} X) \otimes(1+\tilde{\eta} X) \cdot \delta^{\times} f$

- $\operatorname{Ker} \Theta=\left(1+\tilde{\eta} I_{B_{0}}\right)^{\times}$. 
This result was proved in a very detailed way in Section 5 of [4] in a more general context of $p$-group schemes, where $p$ is any prime number. Note that the case $p=2$ is slightly easier to obtain.

If $\tilde{\eta} \notin O_{0}^{*}$ (i.e. if $\mu_{\eta}$ is not multiplicative) then obviously $f \in 1+I_{\bar{B}}^{l o c}$. If $\tilde{\eta} \in O_{0}^{*}$ then we can always multiply $f$ by a suitable element from $\left(1+I_{B^{e t}}\right)^{\times} \subset\left(1+I_{B}\right)^{\times}$to assume again that $f \in 1+I_{\bar{B}}^{l o c}$. This allows us to replace in the above description of $\operatorname{Ext}_{\mathrm{Gr}_{O_{0}}}\left(H_{0}, \mu_{\eta}\right)$, the multiplicative group $\left(1+I_{\bar{B}}\right)^{\times}$by $\mathrm{m}\left(I_{\bar{B}}\right)=I_{\bar{B}}^{\text {loc }}$ with the Lubin-Tate group law from Subsection 2.2.3.

More precisely, let $\delta_{L T}: I_{\bar{B}}^{\text {loc }} \longrightarrow I_{\bar{B} \otimes \bar{B}}^{\text {loc }}$ and $[2]: I_{\bar{B}}^{\text {loc }} \longrightarrow I_{\bar{B}}^{\text {loc }}$ be such that for any $f \in I_{\bar{B}}^{\text {loc }}$,

$$
-\delta_{L T}(f)=[\Delta(f)]-[f \otimes 1]-[1 \otimes f] ;
$$

$-[2](f)=[f]+[f]$.

Let $\mathcal{H}_{L T}=\mathcal{H}_{L T}\left(H_{0}, \mu_{\eta}\right)$ be the subgroup of $I_{\bar{B}}^{\text {loc }}$ (with respect to the Lubin-Tate group law) consisting of $f \in I_{\bar{B}}^{\text {loc }}$ such that $[2](f) \in \tilde{\eta}^{2} I_{B_{0}}$ and $\delta_{L T}(f) \in \tilde{\eta} I_{B_{0} \otimes B_{0}}$. Let $\Theta_{L T}: \mathcal{H}_{L T} \longrightarrow \operatorname{Ext}_{\mathrm{Gr}_{O_{0}}}\left(H_{0}, \mu_{\eta}\right)$ be such that for all $f \in \mathcal{H}_{L T}, \Theta_{L T}(f)=\Theta(E(f))$, where $E(X)=\exp \left(l_{L T}(X)\right)$ is the Artin-Hasse exponential. Our description of $\operatorname{Ext}_{\operatorname{Gr}_{O_{0}}}\left(H_{0}, \mu_{\eta}\right)$ will be used below in the following form.

Proposition 3.2. $\Theta_{L T}$ is a group epimorphism and its kernel equals $\mathcal{H}_{L T} \cap\left(\tilde{\eta} I_{B_{0}}\right)=\left(\tilde{\eta} I_{B_{0}}\right)^{l o c}$.

3.4. Main Lemma. In next subsections we work systematically with the Lubin-Tate group law from Subsection 2.2.3. We always bear in mind the following agreement: if, say, $a \in I_{B}$ appears in the form $[a]$ then $a$ is assumed automatically to be an element of $I_{B}^{l o c}$, that is the corresponding result of the Lubin-Tate addition is automatically well-defined.

Recall that we use the multi-indices $\underline{i}=\left(i_{1}, \ldots, i_{u}\right)$, where all coordinates of the vector $\underline{i}$ belong to $\{0,1\}$. We shall use such indices for the abbreviation $X^{\underline{i}}:=X_{1}^{i_{1}} \ldots X_{u}^{i_{u}}$, especially, when $r(\underline{i}):=i_{1}+\cdots+i_{u} \geqslant 2$. If $r(\underline{i})=1$ then the multi-index $\underline{i}$ appears just as index $j, 1 \leqslant j \leqslant u$, such that $\underline{i}=\left(\delta_{1 j}, \ldots, \delta_{u j}\right)$ (where $\delta$ is the Kronecker symbol).

The following statement is very similar to the statement appeared in Subsection 6.1 of [4] as Main Lemma.

Lemma 3.3 (Main Lemma). If $f \in \mathcal{H}_{L T}=\mathcal{H}_{L T}\left(H_{0}, \mu_{\eta}\right)$ then there are:

$-f_{0} \in \tilde{\eta} I_{B}$

- for $1 \leqslant i \leqslant u, o_{i 0}^{\prime} \in O$ and $o_{i 1}^{\prime} \in \pi^{\prime} O$;

- for all multi-indices $\underline{i}, D_{\underline{i}} \in \bar{O}$, 
such that $o_{i 0}^{\prime 2}, o_{i 1}^{\prime 2} \in \tilde{\eta} O, D_{i} X_{i} \in I_{\bar{B}}(2)^{l o c}, D_{\underline{i}} X^{\underline{i}} \in I_{\bar{B}}^{l o c}$ and

$$
f=\left[f_{0}\right]+\sum_{1 \leqslant i \leqslant u}\left(\left[o_{i 0}^{\prime} X_{i}\right]+\left[o_{i 1}^{\prime} X_{i}\right]+\left[D_{i} X_{i}\right]\right)+\left[2 \sum_{r(\underline{i}) \geqslant 2} D_{\underline{i}} X^{\underline{i}}\right]
$$

Remark. If $\tilde{\eta} \in O_{0}^{*}$ we can always assume that all $o_{i 0}^{\prime}=0$, because all $o_{i 0}^{\prime} X_{i}$ can disappear by contributing to $f_{0}$. In other words, we assume that $o_{i 0}^{\prime 2}, o_{i 1}^{\prime 2} \in(\tilde{\eta} O)^{l o c}$, i.e. these elements belong to $\tilde{\eta} O$ if $1 \leqslant i \leqslant u_{0}$ and belong to the maximal ideal $\mathrm{m}$ of $O$, otherwise.

The proof of this Lemma uses the auxiliary statements from Subsection 3.6 and will be given in Subsection 3.7 below. This is a simplified version of the proof of Main Lemma in [4], where we studied the group schemes of period $p>2$. As a matter of fact, this simplified version works equally well also in the case $p>2$.

3.5. Basic strategy II. Via Main Lemma we shall prove below the existence of $\mathcal{M}^{\prime} \in \mathrm{MF}_{S^{\prime}}^{2 e}$ such that $G^{\prime} \simeq \mathcal{G}_{O^{\prime}}\left(\mathcal{M}^{\prime}\right)$. The description of $G_{0}$ as an element of $\operatorname{Ext}_{\mathrm{Gr}_{O_{0}}}\left(H_{0}, \mu_{\eta}\right)$ from Subsection 3.3 is given in terms of Kummer's theory and, therefore, is of multiplicative nature. On the other hand, the construction of the algebra of $\mathcal{G}_{O^{\prime}}\left(\mathcal{M}^{\prime}\right)$ as extension of $B^{\prime}=B \otimes_{O} O^{\prime}$ should be given (by the definition of $\mathcal{G}_{O^{\prime}}$ ) in additive terms. Therefore, the description of $A\left(G_{0}\right)$ as an extension of $B_{0}=A\left(H_{0}\right)$ in terms of the Lubin-Tate group is a natural step towards presentation of $G \otimes_{O} O^{\prime}$ in the form $\mathcal{G}_{O^{\prime}}\left(\mathcal{M}^{\prime}\right)$.

If $G_{0}$ is given via $f \in \mathcal{H}_{L T}\left(H_{0}, \mu_{\eta}\right)$ then $A_{0}=B_{0}[Y]$ with equation for $Y$ coming from the relation $2 l_{L T}(\tilde{\eta} Y)=l_{L T}(f)$ in $A_{0} \otimes_{O_{0}} K_{0}$. If $\mu_{\eta}$ is multiplicative, e.g. $\tilde{\eta}=1$, then it is much easier to obtain an "integral" version of the above relation. The left-hand side $2 l_{L T}(Y)=$ $2 Y+Y^{2}+2\left(Y^{2} / 2\right)^{2}+\ldots$ looks nicely related to operations in filtered modules of the form $\iota_{\mathcal{M}}(\mathcal{M})$. As for the right-hand side, we need it to belong to $\iota_{\mathcal{N}}(\mathcal{N})$, i.e. to be congruent to a linear combination of all $X_{i}$. By Main Lemma after replacing $f$ by $\tilde{f}=[f]-\left[f_{0}\right], l_{L T}(\tilde{f})$ is a linear combination of all $l_{L T}\left(o_{i 0}^{\prime} X_{i}\right), l_{L T}\left(o_{i 1}^{\prime} X_{i}\right)$ and $l_{L T}\left(D_{i} X_{i}\right)$ modulo $2 I_{\bar{B}}$. First two logarithms can give non-trivial denominators but in $A^{\prime}=$ $A \otimes_{O} O^{\prime}$ we can consider the element $[g]=[\tilde{f}]-\sum_{i}\left(\left[o_{i 0}^{\prime} X_{i}\right]+\left[o_{i 1}^{\prime} X_{i}\right]\right)$ and because all $D_{i} X_{i} \in I_{\bar{B}}(2), l_{L T}(g)$ is an $O^{\prime}$-linear combination of all $X_{1}, \ldots, X_{u}$ modulo $J_{B^{\prime}}$, i.e. gives already an element of $\iota_{\mathcal{N}}(\mathcal{N}) \otimes_{O} O^{\prime}$.

If $\mu_{\eta}$ is not multiplicative the calculations should be more precise because of the extra factor $\tilde{\eta}$. (Here we can see a crucial difference with the case $p>2$, where ideals of the form $(p / \tilde{\eta}) I_{B}$ are still $D P$-ideals.) In particular, we can't ignore the quadratic forms in $X_{i}$ coming from the third term $\left(D_{i} X_{i}\right)^{4} / 4$ of the expansion of $l_{L T}\left(D_{i} X_{i}\right)$ and the second term $g^{2}$ in $l_{L T}(2 g)$, where $g=\sum_{\underline{i}} D_{\underline{i}} X^{\underline{i}}$. The elaboration of Main Lemma from Subsection 3.8 relates these quadratic forms and allows 
us to prove in Subsection 3.9 that they, as a matter of fact, kill oneanother. This provides us in Subsection 3.11 with explicit construction of $\mathcal{M}^{\prime} \in \mathrm{MF}_{S^{\prime}}^{2 e}$ and the existence of $\mathcal{M} \in \mathrm{MF}_{S}^{e}$ such that $\mathcal{M}^{\prime}=\mathcal{M} \otimes_{S} S^{\prime}$. A formal verification that $G \simeq \mathcal{G}_{O}(\mathcal{M})$ is done in Subsection 3.12 .

3.6. Auxiliary statements. Follow Subsection 3.3 of [4] to introduce the ideals $I_{B}(\alpha)$ and $I_{B}(\alpha)^{l o c}$ in $B$, where $\alpha \in O$. Recall that any $a \in I_{B}$ can be uniquely written as $a=\sum_{\underline{\underline{i}}} o_{\underline{i}}(a) X^{\underline{i}}$ with the coefficients $o_{\underline{i}}=o_{\underline{i}}(a) \in O$.

Definition. For any $\alpha \in O$, set

a) $I_{B}(\alpha):=\left\{a \in I_{B} \mid\right.$ all $\left.\left(o_{\underline{i}}(a) X \underline{i}\right)^{2} \in \alpha I_{B}\right\}$

b) $I_{B}(\alpha)^{l o c}:=\left\{a \in I_{B} \mid\right.$ all $\left.\left(o_{\underline{i}}(a) X^{\underline{i}}\right)^{2} \in \alpha I_{B}^{l o c}\right\}$.

Note (use property (C) from Subsection 1.9.1), that for any $1 \leqslant i \leqslant$ $u_{0}, X_{i} \in I_{B}\left(\eta_{i}\right)^{l o c}$ but for $u_{0}<i \leqslant u, X_{i} \notin I_{B}\left(\eta_{i}\right)^{l o c}=I_{B}^{l o c}$. In addition, for arbitrary $\alpha \in O$, the $O$-modules $I_{B}(\alpha)$ and $I_{B}(\alpha)^{l o c}$ depend generally on the above chosen special construction of $B$. Nevertheless, one can verify that:

- for any $\alpha \in O, I_{B}(\alpha)$ and $I_{B}(\alpha)^{l o c}$ are ideals in $B$;

- for $\alpha \mid 2$, we have $I_{B}(\alpha)=\left\{a \in I_{B} \mid a^{2} \in \alpha I_{B}\right\}$ and, similarly, $I_{B}(\alpha)^{l o c}=\left\{a \in I_{B} \mid a^{2} \in \alpha I_{B}^{l o c}\right\}$.

For obvious reasons, the above ideals $I=I_{B}(\alpha)$ and $I=I_{B}(\alpha)^{l o c}$ satisfy property (1.5) from Subsection 1.9.2.

Remark. a) For any $\alpha \in \bar{O}$ we shall denote below by $I_{\bar{B}}(\alpha)$ and $I_{\bar{B}}(\alpha)^{l o c}$ the similar ideals of the $\bar{O}$-algebra $\bar{B}=B \otimes_{O} \bar{O}$. Clearly, they also satisfy property (1.5).

b) Using the special basis $\left\{X^{\underline{i}_{1}} \otimes 1,1 \otimes X^{\underline{i}_{2}}, X^{\underline{i}_{1}} \otimes X^{\underline{i}_{2}} \mid \underline{i}_{1}, \underline{i}_{2}\right\}$ of $I_{B \otimes B}$ and $I_{\bar{B} \otimes \bar{B}}$ we can introduce similarly the ideals $I_{B \otimes B}(\alpha)$ and $I_{\bar{B} \otimes \bar{B}}(\alpha)$.

The following lemmas admit straightforward proofs and are quite analogous to the lemmas from [4] Subsection 6.2, Lemmas 6.2-6.6.

Lemma 3.4. Suppose $C_{1}, \ldots, C_{u} \in \bar{O}, g \in I_{\bar{B}}^{\text {loc }}, \beta_{0} \in \overline{\mathrm{m}}$ and $g \equiv$ $\sum_{i}\left[C_{i} X_{i}\right] \bmod \left(I_{\bar{B}}\left(\beta_{0}\right)^{l o c}+\overline{\mathcal{I}}\right)$, where $\overline{\mathcal{I}} \subset I_{\bar{B}}^{\text {loc }}$ is an ideal satisfying condition (1.5) from Subsection 1.9.2. Then

$$
g \equiv \sum_{1 \leqslant i \leqslant u}\left[C_{i}^{\prime} X_{i}\right]+\left[\sum_{r(\underline{i}) \geqslant 2} C_{\underline{i}}^{\prime} X^{\underline{i}}\right] \bmod \overline{\mathcal{I}}
$$

with all $C_{i}^{\prime}, C_{\underline{i}}^{\prime} \in \bar{O}, C_{i} X_{i} \equiv C_{i}^{\prime} X_{i} \bmod I_{\bar{B}}\left(\beta_{0}\right)^{l o c}$ and $C_{\underline{i}} X^{\underline{i}} \in I_{\bar{B}}\left(\beta_{0}\right)^{l o c}$. 
Lemma 3.5. Suppose l equals either 0 or 1.

a) If $o_{1}^{\prime}, o_{2}^{\prime} \in \pi^{\prime l} O$ are such that $o_{1}^{\prime 2}, o_{2}^{\prime 2} \in \tilde{\eta} O$, then for any $a \in I_{B}$, $\left[o_{1}^{\prime} a\right]+\left[o_{2}^{\prime} a\right]-\left[\left(o_{1}^{\prime}+o_{2}^{\prime}\right) a\right] \in \tilde{\eta} I_{B}$

b) If $o^{\prime} \in \pi^{\prime l} O, o^{\prime 2} \in \tilde{\eta} O$ and $a_{1}, a_{2} \in I_{B}$ then $\left[o^{\prime} a_{1}\right]+\left[o^{\prime} a_{2}\right]-\left[o^{\prime}\left(a_{1}+a_{2}\right)\right] \in \tilde{\eta} I_{B}$.

Remark. a) When proving Lemma 3.4 use first remark from Subsection 1.9.2, b) note the following special cases of above Lemma 3.5:

$-\left[o^{\prime} a\right]+\left[-o^{\prime} a\right] \in \tilde{\eta} I_{B}$

$-\left[\delta_{L T}\left(o^{\prime} X_{i}\right)\right]-\left[o^{\prime} j_{i}\right] \in \tilde{\eta} I_{B \otimes B}$.

Lemma 3.6. If $C \in \bar{O}, \alpha_{1} \in \overline{\mathrm{m}}$ and $C X_{i} \in I_{\bar{B}}\left(\alpha_{1}\right)^{\text {loc }}$ then

$$
\delta_{L T}\left(C X_{i}\right) \equiv C^{2} X_{i} \otimes X_{i} \bmod I_{\bar{B}}\left(\alpha_{1}^{4}\right)^{l o c}+\overline{\mathcal{J}},
$$

where $\overline{\mathcal{J}}$ is the ideal defined in the end of Subsection 1.9.2.

Remark. Note that if $C X_{i} \in I_{\bar{B}}(2)^{l o c}$ then $C^{2} X_{i} \otimes X_{i} \in \overline{\mathcal{J}}$.

Proof. From the definition of the Lubin-Tate group law, cf. Subsection 2.2 .3 it follows that

$$
\begin{aligned}
& \delta_{L T}\left(C X_{i}\right)=\left[C j_{i}\right]-\left[-C^{2}\left(X_{i} \otimes 1+1 \otimes X_{i}\right) j_{i}\right]-\left[-C^{2} X_{i} \otimes X_{i}\right] \\
& -\sum_{n \geqslant 2}\left[C^{2^{n}} P_{n}\left(X_{i} \otimes 1+1 \otimes X_{i}, j_{i}\right)\right]-\sum_{n \geqslant 2}\left[C^{2^{n}} P_{n}\left(X_{i} \otimes 1,1 \otimes X_{i}\right)\right]
\end{aligned}
$$

We know that for $1 \leqslant i \leqslant u_{0}, j_{i} \in \overline{\mathcal{J}}$ and if $u_{0}<i \leqslant u$ then $C \in \overline{\mathrm{m}}$ and again $C j_{i} \in \overline{\mathcal{J}}$. Therefore, the first, second and forth terms of the right-hand side of (3.1) belong to $\overline{\mathcal{J}}$. As for the last term of that formula it remains to note that $\left(I_{\bar{B}}\left(\alpha_{1}\right)^{l o c}\right)^{4} \subset I_{\bar{B}}\left(\alpha_{1}^{4}\right)^{l o c}$.

3.7. Proof of Main Lemma. Prove that for any $\alpha \in \overline{\mathrm{m}}$, one has

$$
f \equiv\left[f_{\alpha}\right]+\sum_{1 \leqslant i \leqslant u}\left(\left[o_{i 0}^{\prime} X_{i}\right]+\left[o_{i 1}^{\prime} X_{i}\right]+\left[D_{i} X_{i}\right]\right) \bmod \left(2 I_{\bar{B}}^{l o c}+I_{\bar{B}}\left(\alpha^{2}\right)^{l o c}\right)
$$

where

- $f_{\alpha} \in \tilde{\eta} I_{B}$

- all $o_{i 0}^{\prime}=o_{i 0}^{\prime}(\alpha) \in O, o_{i 1}^{\prime}=o_{i 1}^{\prime}(\alpha) \in \pi^{\prime} O$ are such that $o_{i 0}^{\prime 2}, o_{i 1}^{\prime 2} \in \tilde{\eta} O$; (If $\tilde{\eta} \in O_{0}^{*}$ then we can assume that all $o_{i 0}^{\prime}=0$.)

- all $D_{i} \in \bar{O}$ are such that $D_{i} X_{i} \in I_{\bar{B}}(\alpha, 2)^{l o c}:=I_{\bar{B}}(\alpha)^{l o c}+I_{\bar{B}}(2)^{l o c}$.

First, there is an $\alpha_{0} \in \mathrm{m}$ such that (3.2) holds for trivial reasons with $\alpha=\alpha_{0}$. Indeed, if $\tilde{\eta} \notin O_{0}^{*}$ then use that $f^{2} \equiv[2](f) \equiv 0 \bmod \tilde{\eta} I_{\bar{B}}$; otherwise, use that $[f]-\left[f_{0}\right] \in \overline{\mathrm{m}} I_{\bar{B}}$, where $f_{0} \in I_{B}$ and $f \equiv f_{0} \bmod \overline{\mathrm{m}} I_{\bar{B}}$.

By induction on $\alpha$ it will be sufficient to prove that if (3.2) holds with $\alpha=\alpha_{1} \in \overline{\mathrm{m}}$ then it also holds with $\alpha=\alpha_{1}^{2}$. 
Apply Lemma 3.4 with $\overline{\mathcal{I}}=2 I_{\bar{B}}^{\text {loc }}$ to obtain

$f=\left[f_{\alpha_{1}}\right]+\sum_{1 \leqslant i \leqslant u}\left(\left[o_{i 0}^{\prime} X_{i}\right]+\left[o_{i 1}^{\prime} X_{i}\right]+\left[D_{i}^{\prime} X_{i}\right]\right)+\left[\sum_{r(\underline{i}) \geqslant 2} D_{\underline{i}}^{\prime} X^{\underline{i}}\right] \bmod 2 I_{\bar{B}}^{l o c}$,

where all $D_{i}^{\prime}, D_{\underline{i}}^{\prime} \in \bar{O}, D_{i}^{\prime} X_{i} \in I_{\bar{B}}\left(\alpha_{1}, 2\right)^{l o c}$ and $D_{\underline{i}}^{\prime} X^{\underline{i}} \in I_{\bar{B}}\left(\alpha_{1}^{2}\right)^{l o c}$.

By Lemma 3.5 all $\delta_{L T}\left(o_{i 0}^{\prime} X_{i}\right), \delta_{L T}\left(o_{i 1}^{\prime} X_{i}\right) \in \tilde{\eta} \tilde{I}_{B \otimes B}+\overline{\mathcal{J}}$. Therefore, the condition $\delta_{L T}(f) \in \tilde{\eta} I_{B_{0} \otimes B_{0}} \subset \tilde{\eta} I_{B \otimes B}$ implies (use Lemma 3.6) that

$$
\sum_{1 \leqslant i \leqslant u} D_{i}^{\prime 2} X_{i} \otimes X_{i}+\sum_{\underline{i}_{1}+\underline{i}_{2}=\underline{i}} D_{\underline{i}}^{\prime} X^{\underline{i}_{1}} \otimes X^{\underline{i}_{2}} \in \tilde{\eta} I_{B \otimes B} \bmod \left(I_{\bar{B} \otimes \bar{B}}\left(\alpha_{1}^{4}\right)^{l o c}+\overline{\mathcal{J}}\right)
$$

(Recall that all multi-indices $\underline{i}, \underline{i}_{1}, \underline{i}_{2}$ are non-zero vectors with coordinates 0 or 1. )

This implies the following properties (cf. first Remark in Subsection 1.9.2):

a) for $1 \leqslant i \leqslant u, D_{i}^{\prime 2} X_{i} \otimes X_{i} \equiv o_{i} X_{i} \otimes X_{i} \bmod \left(I_{\bar{B} \otimes \bar{B}}\left(\alpha_{2}^{2}\right)^{l o c}+\overline{\mathcal{J}}\right)$, where $o_{i} \in \tilde{\eta} O$;

b) if $r(\underline{i}) \geqslant 2$ then $D_{\underline{\underline{i}}}^{\prime} X^{\underline{i}_{1}} \otimes X^{\underline{i}_{2}} \equiv o_{\underline{\underline{i}}} X^{\underline{i}_{1}} \otimes X^{\underline{i}_{2}} \bmod \left(I_{\bar{B} \otimes \bar{B}}\left(\alpha_{2}^{2}\right)^{l o c}+\overline{\mathcal{J}}\right)$, where $o_{\underline{i}} \in \tilde{\eta} O$.

Consider the morphism of multiplication $m: \bar{B} \otimes \bar{B} \longrightarrow \bar{B}$. Then $m\left(I_{\bar{B} \otimes \bar{B}}\left(\alpha_{2}^{2}\right)^{l o c}\right)=I_{\bar{B}}\left(\alpha_{2}^{2}\right)^{l o c}, m(\overline{\mathcal{J}})=2 I_{\bar{B}}^{l o c}$. Therefore, b) implies that $\left(D_{\underline{i}}^{\prime}-o_{\underline{i}}\right) X^{\underline{i}} \in I_{\bar{B} \otimes \bar{B}}\left(\alpha_{2}^{2}\right)^{l o c}+2 I_{\bar{B}}^{\text {loc }}$ and the last summand in (3.3) disappears modulo $I_{\bar{B}}\left(\alpha_{2}^{2}\right)^{l o c}$ by contributing to the corresponding $f_{\alpha_{2}} \in \tilde{\eta} I_{B}$. If $2 \mid \alpha_{1}$ formula (3.2) has been already proved for $\alpha=\alpha_{2}$ because we don't need to change the terms $D_{i}^{\prime} X_{i}$.

If $\alpha_{1} \mid 2$ we should continue with property a). If $D_{i}^{\prime} X_{i} \otimes X_{i} \in \overline{\mathcal{J}}$ keep $D_{i}^{\prime}$ the same. Otherwise, by first Remark from Subsection 1.9.2 we can assume that $D_{i}^{\prime} X_{i} \otimes X_{i} \in I_{\bar{B} \otimes \bar{B}}\left(\alpha_{2}^{2}\right)^{l o c}$. Consider the elements $o_{i 0}^{\prime \prime} \in O$ and $o_{i 1}^{\prime \prime} \in \pi^{\prime} O$ such that $o_{i} \equiv o_{i 0}^{\prime \prime 2}+o_{i 1}^{\prime \prime 2} \bmod 2 o_{i} \pi$ and $o_{i 0}^{\prime \prime 2}, o_{i 1}^{\prime \prime 2} \equiv 0 \bmod o_{i}$.

Lemma 3.7. $\left[D_{i}^{\prime} X_{i}\right]+\left[-o_{i 0}^{\prime \prime} X_{i}\right]+\left[-o_{i 1}^{\prime \prime} X_{i}\right] \equiv \sum_{j}\left[D_{i j}^{\prime} X_{j}\right] \bmod I_{\bar{B}}\left(\alpha_{2}^{2}\right)^{l o c}$, where all $D_{i j}^{\prime} \in \bar{O}$ and $D_{i j}^{\prime} X_{j} \in I_{\bar{B}}\left(\alpha_{2}^{2}\right)^{l o c}$.

Proof. Suppose $1 \leqslant i \leqslant u_{0}$. In this case $X_{i}^{2} \in I_{B}\left(\eta_{i}\right)^{\text {loc }}$ and property a) means that $\left(D_{i}^{\prime 2}-o_{i}\right) \eta_{i} \in \alpha_{2} \bar{O}$. Therefore (use that $\left.\alpha_{2} \mid 4\right),\left(D_{i}^{\prime}-o_{i 0}^{\prime \prime}-\right.$ $\left.o_{i 1}^{\prime \prime}\right)^{2} \eta_{i} \in \alpha_{2} \bar{O}$, i.e. $\left(D_{i}^{\prime}-o_{i 0}^{\prime \prime}-o_{i 1}^{\prime \prime}\right) X_{i} \in I_{\bar{B}}\left(\alpha_{2}\right)^{l o c}$. Also, $0 \equiv D_{i}^{\prime 2} \eta_{i} \equiv$ $o_{i} \eta_{i} \equiv o_{i 0}^{\prime \prime 2} \eta_{i} \equiv o_{i 1}^{\prime \prime 2} \eta_{i} \bmod \alpha_{1} \bar{O}$, i.e. $o_{i 0}^{\prime \prime} X_{i}, o_{i 1}^{\prime \prime} X_{i} \in I_{\bar{B}}\left(\alpha_{1}\right)^{l o c}$.

Suppose $u_{0}<i \leqslant u$. In this case $\eta_{i} \in O^{*}, D_{i} \in \overline{\mathrm{m}}$ and $X_{i}, X_{i}^{2} \in I_{B}^{e t}$. The relation $\left(D_{i}^{\prime 2}-o_{i}\right) X_{i} \otimes X_{i} \in I_{\bar{B}}\left(\alpha_{2}^{2}\right)^{l o c}$ means that $\left(D_{i}^{\prime 2}-o_{i}\right) \eta_{i} \in \alpha_{2} \overline{\mathrm{m}}$, $D_{i}^{\prime 2} \eta_{i} \equiv\left(o_{i 0}^{\prime \prime 2}+o_{i 1}^{\prime \prime 2}\right) \eta_{i} \bmod \alpha_{2} \overline{\mathrm{m}},\left(D_{i}^{\prime}-o_{i 0}^{\prime \prime}-o_{i 1}^{\prime \prime}\right)^{2} \eta_{i} \in \alpha_{2} \overline{\mathrm{m}}$ and again $\left(D_{i}-o_{i 0}^{\prime \prime}-o_{i 1}^{\prime \prime}\right) X_{i} \in I_{\bar{B}}\left(\alpha_{2}^{2}\right)^{l o c}$. Similarly, $D_{i}^{\prime} X_{i} \in I_{\bar{B}}\left(\alpha_{1}\right)^{l o c}$ means that $0 \equiv D_{i}^{\prime 2} \eta_{i} \equiv o_{i} \eta_{i} \equiv o_{i 0}^{\prime \prime 2} \eta_{i} \equiv o_{i 1}^{\prime \prime 2} \eta_{i} \bmod \alpha_{1} \overline{\mathrm{m}}$, i.e. $o_{i 0}^{\prime \prime} X_{i}, o_{i 1}^{\prime \prime} X_{i} \in I_{\bar{B}}\left(\alpha_{1}\right)^{l o c}$. 
Now for any $1 \leqslant i \leqslant u$,

$$
\begin{aligned}
& {\left[D_{i}^{\prime} X_{i}\right]+\left[-o_{i 0}^{\prime \prime} X_{i}\right]+\left[-o_{i 1}^{\prime \prime} X_{i}\right] \equiv\left[\left(D_{i}^{\prime}-o_{i 0}^{\prime \prime}-o_{i 1}^{\prime \prime}\right) X_{i}\right]} \\
& -\left[D_{i}^{\prime} o_{i 0}^{\prime \prime} X_{i}^{2}\right]-\left[D_{i}^{\prime} o_{i 1}^{\prime \prime} X_{i}^{2}\right]-\left[-o_{i 0}^{\prime \prime} o_{i 1}^{\prime \prime} X_{i}^{2}\right] \bmod I_{\bar{B}}\left(\alpha_{2}^{2}\right)^{l o c}
\end{aligned}
$$

where all terms in the right-hand side belong to $\left(I_{\bar{B}}\left(\alpha_{1}\right)^{l o c}\right)^{2} \subset I_{\bar{B}}\left(\alpha_{2}\right)^{l o c}$. Thus the right-hand side is congruent modulo $I_{\bar{B}}\left(\alpha_{2}^{2}\right)^{l o c}$ to $\sum_{j}\left[D_{i j}^{\prime} X_{j}\right]$ with all $D_{i j}^{\prime} \in \bar{O}$ and $D_{i j}^{\prime} X_{j} \in I_{\bar{B}}\left(\alpha_{2}\right)^{l o c}$. Lemma 3.7 is proved.

Finally, we finish the proof of formula (3.2) with $\alpha=\alpha_{2}$ by noting that (use Lemma 3.5)

where $\tilde{a} \in \tilde{\eta} I_{B}$.

$$
\begin{gathered}
\sum_{i}\left(\left[o_{i 0}^{\prime} X_{i}\right]+\left[o_{i 1}^{\prime} X_{i}\right]\right)-\sum_{i}\left(\left[-o_{i 0}^{\prime \prime} X_{i}\right]+\left[-o_{i 1}^{\prime \prime} X_{i}\right]\right)= \\
{[\tilde{a}]+\sum_{i}\left(\left[\left(o_{i 0}^{\prime}+o_{i 0}^{\prime \prime}\right) X_{i}\right]+\left[\left(o_{i 1}^{\prime}+o_{i 1}^{\prime \prime}\right) X_{i}\right]\right),}
\end{gathered}
$$

Clearly, there is $\alpha \in \overline{\mathrm{m}}$ such that $I_{\bar{B}}\left(\alpha^{2}\right)^{l o c} \subset 2 I_{\bar{B}}^{\text {loc }}$. It remains to apply Lemma 3.4 with $\overline{\mathcal{I}}=0$ to finish the proof of Main Lemma.

3.8. Elaboration of Main Lemma. Let $D=C^{-1}=\left(d_{i j}\right)$ and for $1 \leqslant i \leqslant u$,

$$
R_{i}=\sum_{\substack{t \\ s_{1}<s_{2}}}\left(X_{s_{1}} \otimes X_{s_{2}}+X_{s_{2}} \otimes X_{s_{1}}\right) c_{s_{1} t} c_{s_{2} t} d_{t i}^{2} \in I_{B \otimes B}
$$

Then property $(\mathbf{C})$ of the matrix $C$ from Subsection 1.9 .1 implies that all $\tilde{\eta}_{i} R_{i} \in I_{B \otimes B}^{l o c}$. Indeed, if $\tilde{\eta}_{i} X_{s_{1}} \otimes X_{s_{2}} \notin I_{B \otimes B}^{l o c}$ then $1 \leqslant i \leqslant u_{0}$ (because $\tilde{\eta}_{i}$ must belong to $O_{0}^{*}$ ) and $u_{0}<s_{1}, s_{2} \leqslant u$ (because $X_{s_{1}}, X_{s_{2}} \notin I_{B}^{l o c}$ ), but then $c_{s_{1} t} d_{t i}, c_{s_{2} t} d_{t i} \equiv 0 \bmod \pi$. Therefore, the system of congruences

$$
\sum_{s} B_{s} c_{s i} \equiv \tilde{\eta}_{i}\left(R_{i}+B_{i}^{2}\right) \bmod I_{B \otimes B}(4)^{l o c}, \quad 1 \leqslant i \leqslant u,
$$

has a unique solution $\left(B_{1}, \ldots, B_{u}\right) \bmod I_{B \otimes B}(4)^{l o c}$ with all $B_{i} \in I_{B \otimes B}^{l o c}$.

We shall use below the following agreement: if $1 \leqslant i \leqslant u$ and $\alpha \in O$ then $I_{B}(\alpha)^{(i)}$ will be equal to $I_{B}(\alpha)^{l o c}$ if $i \leqslant u_{0}$ and to $I_{B}(\alpha)$ if $i>u_{0}$. Same agreement will be used for similar ideals $I_{B \otimes B}(\alpha), I_{\bar{B}}(\alpha)$, etc.

Lemma 3.8. With above notation one has

$$
\begin{aligned}
j_{i} \equiv \sum_{t} \tilde{\eta}_{t} X_{t} \otimes & X_{t} d_{t i}+2 B_{i}+\sum_{t, u} \tilde{\eta}_{u}\left(X_{u} \otimes X_{u}\right) d_{u t}\left(\tilde{\eta}_{t} X_{t} \otimes 1+1 \otimes \tilde{\eta}_{t} X_{t}\right) d_{t i} \\
& +2 \sum_{t}\left(\tilde{\eta}_{t} X_{t} \otimes 1+1 \otimes \tilde{\eta}_{t} X_{t}\right) B_{t} d_{t i} \bmod I_{B \otimes B}(16)^{(i)}
\end{aligned}
$$

Proof. It will be sufficient to verify by direct calculations that the righthand sides of above congruences give solutions of equalities (1.3) modulo $I_{B \otimes B}(16)^{(i)}$. (When calculating use that the first two summands belong to $I_{B \otimes B}(4)^{(t)}$ and the last two ones - to $I_{B \otimes B}(8)^{(t)}$.) 

form

One can easily see that the above elements $B_{i} \in I_{B \otimes B}^{l o c}$ appear in the

$$
B_{i} \equiv \sum_{s_{1}<s_{2}} \gamma_{s_{1} s_{2} i}\left(X_{s_{1}} \otimes X_{s_{2}}+X_{s_{2}} \otimes X_{s_{1}}\right) \bmod I_{B \otimes B}(4)^{l o c}
$$

where all $\gamma_{s_{1} s_{2} i} \in O$. Introduce the elements

$$
\widetilde{R}_{i}=\sum_{\substack{t \\ s_{1}<s_{2}}} X_{s_{1}} X_{s_{2}} c_{s_{1} t} c_{s_{2} t} d_{t i}^{2}, \quad \widetilde{B}_{i}=\sum_{s_{1}<s_{2}} \gamma_{s_{1} s_{2} i} X_{s_{1}} X_{s_{2}}
$$

Then

- $\left(\widetilde{B}_{1}, \ldots, \widetilde{B}_{u}\right) \bmod I_{B}(4)^{l o c}$ is a unique solution in $I_{B}^{l o c} \bmod I_{B}(4)^{l o c}$ of the congruences $\sum_{s} \widetilde{B}_{s} c_{s i} \equiv \tilde{\eta}_{i}\left(\widetilde{R}_{i}+\widetilde{B}_{i}^{2}\right) \bmod I_{B}(4)^{l o c}$;

- for all $i, \delta^{+}\left(\widetilde{B}_{i}\right) \equiv B_{i} \bmod I_{B \otimes B}(4)^{l o c}$.

Now we can state the following elaboration of Main Lemma.

Proposition 3.9. In Lemma 3.3 the elements $D_{i} \in \bar{O}, 1 \leqslant i \leqslant u$, and $g:=\sum_{r(\underline{i}) \geqslant 2} D_{\underline{i}} X^{\underline{i}} \in I_{\bar{B} \otimes \bar{B}}$ can be taken in such a way that

a) $\left(D_{i}^{2}+\sum_{s}\left(o_{s 0}^{\prime}+o_{s 1}^{\prime}+D_{s}\right) d_{i s} \tilde{\eta}_{i}-\tilde{o}_{i}\right) X_{i}^{2} \in 4 I_{\bar{B}}$, where $1 \leqslant i \leqslant u$ and all $\tilde{o}_{i} \in \tilde{\eta} O$;

b) $g \equiv \sum_{i}\left(o_{i 0}^{\prime}+o_{i 1}^{\prime}+D_{i}\right) \widetilde{B}_{i} \bmod I_{\bar{B}}(4)^{l o c}$.

Remark. Part a) implies that all $\tilde{o}_{i} \equiv 0 \bmod \tilde{\eta}_{i}$ and if $u_{0}<t \leqslant u$ then $\tilde{o}_{i} \equiv 0 \bmod \pi$. In other words, all $\tilde{o}_{i} \in\left(\tilde{\eta}_{i} O\right)^{l o c}$.

Proof. Let $\tilde{f}:=[f]-\left[f_{0}\right]$. Then

$$
\begin{aligned}
& \delta_{L T}(\tilde{f}) \equiv \sum_{i}\left(o_{i 0}^{\prime}+o_{i 1}^{\prime}+D_{i}\right) j_{i}+\sum_{i} D_{i}^{2} X_{i} \otimes X_{i} \\
& \quad+\sum_{i} D_{i}^{2}\left(X_{i} \otimes 1+1 \otimes X_{i}\right) j_{i}+2 \delta^{+}(g) \bmod I_{\bar{B} \otimes \bar{B}}(16)^{l o c} .
\end{aligned}
$$

For $1 \leqslant t \leqslant u$, let $s(t)=\sum_{i}\left(o_{i 0}^{\prime}+o_{i 1}^{\prime}+D_{i}\right) d_{t i} \tilde{\eta}_{t}$. Then using the explicit formulas for $j_{i} \bmod I_{B \otimes B}(16)^{(i)}, 1 \leqslant i \leqslant u$, from Lemma 3.8 we obtain that $\delta_{L T}(\tilde{f})$ is congruent modulo $I_{\bar{B} \otimes \bar{B}}(16)^{l o c}$ to

$\sum_{t}\left(D_{t}^{2}+s(t)\right) X_{t} \otimes X_{t}+2 \sum_{t}\left(o_{t 0}^{\prime}+o_{t 1}^{\prime}+D_{t}\right) B_{t}+$

$\sum_{t}\left(D_{t}^{2}+s(t)\right)\left(X_{t} \otimes 1+1 \otimes X_{t}\right)\left(2 B_{t}+\sum_{u} d_{u t} \tilde{\eta}_{u} X_{u} \otimes X_{u}\right)+2 \delta^{+}(g)$.

Follow the coefficient for $X_{i} \otimes X_{i}, 1 \leqslant i \leqslant u$.

Verify that only the first sum contributes to this coefficient.

Indeed, the second sum does not contribute because $B_{t} \bmod I_{B \otimes B}(4)^{l o c}$ is a linear combination of the terms $X_{s_{1}} \otimes X_{s_{2}}$ with $s_{1} \neq s_{2}$. The remaining big sum also does not contribute modulo $4 I_{\bar{B} \otimes \bar{B}}^{\text {loc }}$ because:

$-2 B_{t}\left(X_{t} \otimes 1\right)$ and $2 B_{t}\left(1 \otimes X_{t}\right)$ contribute in the same way; 
- for similar reason it will be sufficient to verify that $X_{i} \otimes X_{i}$ can appear in $\left(\tilde{\eta}_{u} X_{u} \otimes X_{u}\right)\left(X_{t} \otimes 1\right)$ with coefficient from $2 O$; but if this coefficient is not 0 then $u=t$ and we can use that $\eta_{u} \tilde{\eta}_{u}=-2$.

So, for $1 \leqslant i \leqslant u,\left(D_{i}^{2}+s(i)\right) X_{i} \otimes X_{i} \in \tilde{\eta} I_{B \otimes B} \bmod I_{\bar{B} \otimes \bar{B}}(16)^{l o c}$. Therefore, there is $\tilde{o}_{i} \in \tilde{\eta} O$ such that $\left(D_{i}^{2}+s(i)-\tilde{o}_{i}\right) \eta_{i}$ belongs to $4 \bar{O}$. This proves part a).

The remaining terms in the relation $\delta_{L T}(\tilde{f}) \in \tilde{\eta} I_{B \otimes B} \bmod I_{\bar{B} \otimes \bar{B}}(16)^{l o c}$ give

$$
\sum_{i}\left(o_{i 0}^{\prime}+o_{i 1}^{\prime}+D_{i}\right) B_{i}+\delta^{+}(g) \in I_{B \otimes B} \bmod I_{\bar{B} \otimes \bar{B}}(4)^{l o c}
$$

(use property a) to eliminate the last big sum).

We know that $h=\sum_{i}\left(o_{i 0}^{\prime}+o_{i 1}^{\prime}+D_{i}\right) \widetilde{B}_{i}+g$ is of the form $\sum_{r(\underline{i}) \geqslant 2} C_{\underline{i}} X^{\underline{i}}$. Therefore, the relation $\delta^{+} h \in I_{B \otimes B} \bmod I_{\bar{B} \otimes \bar{B}}(4)^{l o c}$ implies that $h \equiv$ $h_{0} \bmod I_{\bar{B}}(4)^{l o c}$ with $h_{0} \in I_{B}$. So, replacing $f_{0}$ by $f_{0}-2 h_{0}$ we obtain property $b)$.

3.9. Explicit calculation of $l_{L T}(f)$. As earlier, $f \in \mathcal{H}_{L T}\left(H_{0}, \mu_{\eta}\right)$ is given via Main Lemma where we can now assume that $D_{1}, \ldots, D_{u} \in \bar{O}$ and $g \in I_{\bar{B}}^{l o c}$ satisfy Proposition 3.9 , We also set $\tilde{f}=[f]-\left[f_{0}\right], L_{\bar{B}}=$ $\sum_{i} \bar{O} X_{i}, L_{\bar{B}}^{\text {loc }}=\sum_{1 \leqslant i \leqslant u_{0}} \bar{O} X_{i}+\sum_{u_{0}<i \leqslant u} \overline{\mathrm{m}} X_{i}$ and define

$$
\left(\tilde{\eta} L_{\bar{B}}\right)^{l o c}=\left\{\begin{array}{ll}
\tilde{\eta} L_{\bar{B}} & \text { if } \tilde{\eta} \notin O^{*} \\
L_{\bar{B}}^{l o c} & \text { if } \tilde{\eta} \in O^{*}
\end{array} \quad\left(\tilde{\eta} I_{\bar{B}}(4)\right)^{l o c}= \begin{cases}\tilde{\eta} I_{\bar{B}}(4) & \text { if } \tilde{\eta} \notin O^{*} \\
I_{\bar{B}}(4)^{l o c} & \text { if } \tilde{\eta} \in O^{*}\end{cases}\right.
$$

For $1 \leqslant i \leqslant u$ and $\tilde{o}_{i} \in O$ from Proposition 3.9, let $o_{i 2}^{\prime} \in O$ and $o_{i 3}^{\prime} \in \pi^{\prime} O$ be such that $\tilde{o}_{i} \equiv o_{i 2}^{\prime 2}+o_{i 3}^{\prime 2} \bmod 2 \tilde{\eta} \pi O$ and $o_{i 2}^{\prime 2}, o_{i 3}^{\prime 2} \in \tilde{o}_{i} O$.

Proposition 3.10. With above notation

$$
l_{L T}(\tilde{f}) \equiv-\sum_{i, l} o_{i l}^{\prime} X_{i}+\sum_{i, l} l_{L T}\left(o_{i l}^{\prime} X_{i}\right) \bmod \left(\left(\tilde{\eta} L_{\bar{B}}\right)^{l o c}+\left(\tilde{\eta} I_{\bar{B}}(4)\right)^{l o c}\right) .
$$

(In both sums $1 \leqslant i \leqslant u$ and $0 \leqslant l \leqslant 3$.)

Proof. Note that (use that all $D_{i} X_{i} \in I_{\bar{B}}(2)$ )

$$
l_{L T}\left(D_{i} X_{i}\right) \equiv D_{i} X_{i}+\frac{D_{i}^{2} X_{i}^{2}}{2}+\left(\frac{D_{i}^{2} X_{i}^{2}}{2}\right)^{2}+2\left(\frac{D_{i}^{2} X_{i}^{2}}{2}\right)^{4} \bmod 4 I_{\bar{B}}
$$

Rewrite property a) of Proposition 3.9 in the following form

$$
\frac{D_{i}^{2} X_{i}^{2}}{2} \equiv \frac{\tilde{o}_{i} X_{i}^{2}}{2}-\sum_{s}\left(o_{s 0}^{\prime}+o_{s 1}^{\prime}+D_{s}\right) d_{i s} \tilde{\eta}_{i} X_{i}^{2} \bmod 2 L_{\bar{B}}
$$

Then

$$
\sum_{i} \frac{D_{i}^{2} X_{i}^{2}}{2} \equiv \sum_{i} \frac{\tilde{o}_{i} X_{i}^{2}}{2}-\sum_{s}\left(o_{s 0}^{\prime}+o_{s 1}^{\prime}+D_{s}\right) X_{s} \bmod 2 L_{\bar{B}}
$$


Property a) also implies that

$$
\left(\frac{D_{i}^{2} X_{i}^{2}}{2}\right)^{2} \equiv\left(\frac{\tilde{o}_{i}^{2}}{\tilde{\eta}_{i}}-\sum_{s}\left(o_{s 0}^{\prime}+o_{s 1}^{\prime}+D_{s}\right) d_{i s}\right)^{2}\left(X_{i}^{2} / \eta_{i}\right)^{2} \bmod 4 I_{\bar{B}}
$$

The first factor in the right-hand side of the last congruence can be written in the form

$$
\left(\frac{o_{i 2}^{\prime 2}}{\tilde{\eta}_{i}}\right)^{2}+\left(\frac{o_{i 3}^{\prime 2}}{\tilde{\eta}_{i}}\right)^{2}+\sum_{s} D_{s}^{2} d_{i s}^{2}+\tilde{\eta} A_{i}
$$

where $A_{i} \in \bar{O}$ and belongs to the maximal ideal $\overline{\mathrm{m}}$ of $\bar{O}$ if $u_{0}<i \leqslant u$. Then

$$
\left(\frac{D_{i}^{2} X_{i}^{2}}{2}\right)^{2} \equiv\left(\frac{o_{i 2}^{\prime 2} X_{i}^{2}}{2}\right)^{2}+\left(\frac{o_{i 3}^{\prime 2} X_{i}^{2}}{2}\right)^{2}+2 \sum_{\substack{s \\ u_{1}<u_{2}}} D_{s}^{2} d_{i s}^{2} X_{u_{1}} X_{u_{2}} c_{u_{1} i} c_{u_{2} i}
$$

modulo $\left(\tilde{\eta} L_{\bar{B}}\right)^{l o c}+2\left(\tilde{\eta} I_{\bar{B}}\right)^{l o c}$, because

$$
\sum_{i, s, u} D_{s}^{2} d_{i s}^{2} X_{u}^{2} c_{u i}^{2} \equiv \sum_{s} D_{s}^{2} X_{s}^{2} \equiv 0 \bmod 2 L_{\bar{B}}
$$

Note also that

$$
2\left(\frac{D_{i}^{2} X_{i}^{2}}{2}\right)^{4}=2\left(\frac{D_{i}^{2} \eta_{i}}{2}\right)^{4}\left(\sum_{s} X_{s}^{2} c_{s i}^{2}+2 \sum_{s_{1}<s_{2}} X_{s_{1}} X_{s_{2}} c_{s_{1} i} c_{s_{2} i}\right)^{2}
$$

belongs to $2 L_{\bar{B}}+4 I_{\bar{B}}$.

Taking above calculations together we obtain

$$
\begin{gathered}
\sum_{i} l_{L T}\left(D_{i} X_{i}\right) \equiv-\sum_{i}\left(o_{i 0}^{\prime}+o_{i 1}^{\prime}\right) X_{i}+\sum_{i, l} \frac{o_{i l}^{\prime 2} X_{i}^{2}}{2}+2 \sum_{s} D_{s}^{2} \widetilde{R}_{s} \equiv \\
-\sum_{i, l} o_{i l}^{\prime} X_{i}+\sum_{i} l_{L T}\left(\left[o_{i 2}^{\prime} X_{i}\right]+\left[o_{i 3}^{\prime} X_{i}\right]\right)+2 \sum_{s} D_{s}^{2} \widetilde{R}_{s}
\end{gathered}
$$

modulo $\left(\tilde{\eta} L_{\bar{B}}\right)^{l o c}+2\left(\tilde{\eta} I_{\bar{B}}\right)^{l o c}$ because all $2\left(o_{i 2}^{\prime 2} X_{i}^{2} / 2\right)^{4}$ and $2\left(o_{i 3}^{\prime 2} X_{i}^{2} / 2\right)^{4}$ belong to $2 L_{\bar{B}}+4 I_{\bar{B}}$.

On the other hand we have:

$g^{2} \equiv \sum_{t} D_{t}^{2} \widetilde{B}_{t}^{2} \bmod \left(\tilde{\eta} I_{\bar{B}}\right)^{l o c}$, use Proposition $\left.3.9 \mathrm{~b}\right) ;$

$\sum_{t} D_{t}^{2} \widetilde{B}_{t}^{2} \equiv \sum_{t, s}\left(D_{t}^{2} / \tilde{\eta}_{t}\right) \widetilde{B}_{s} c_{s t}-\sum_{t} D_{t}^{2} \widetilde{R}_{t} \bmod I_{\bar{B}}(4)^{l o c}$, cf. Subsection 3.9.

$D_{t}^{2} / \tilde{\eta}_{t} \equiv-\sum_{s}\left(o_{s 0}^{\prime}+o_{s 1}^{\prime}+D_{s}\right) d_{i s} \bmod \tilde{\eta} \bar{O}$, use Proposition $\left.3.9 \mathrm{a}\right)$.

Therefore,

$$
l_{L T}(2 g) \equiv 2\left(g^{2}+g\right) \equiv-2 \sum_{t} D_{t}^{2} \widetilde{R}_{t} \bmod \left(\tilde{\eta} I_{\bar{B}}(4)\right)^{l o c}
$$

because $2\left(\tilde{\eta} I_{\bar{B}}\right)^{l o c}, 2 I_{\bar{B}}(4)^{l o c}, 2 \tilde{\eta} I_{B \otimes B}^{l o c}$ are contained in $\left(\tilde{\eta} I_{\bar{B}}(4)\right)^{l o c}$.

The Proposition is proved. 
3.10. Special element $h \in I_{\bar{B}}$ and its properties. With above notation set

$$
h=[\tilde{f}]-\sum_{i, l}\left[o_{i l}^{\prime} X_{i}\right]
$$

Note that $[2](h)=[2](f)-[2]\left(f_{0}\right)-\sum_{i, l}[2]\left(o_{i l}^{\prime} X_{i}\right) \in I_{B^{\prime}} \subset I_{\bar{B}}$ and, therefore, $l_{L T}(h)=(1 / 2) l_{L T}([2] h) \in I_{B^{\prime}} \otimes_{O} K$. Then by Proposition 3.10

$$
l_{L T}(h) \equiv \tilde{\eta}^{\prime} l_{0} \bmod \left(\tilde{\eta} I_{B^{\prime}}(4)\right)^{l o c},
$$

where $l_{0}=\sum_{i} a_{i} X_{i}$ with $a_{i} \in O^{\prime}$ and one has

$$
\tilde{\eta}^{\prime} a_{i} \equiv-\sum_{l} o_{i l}^{\prime} \bmod \tilde{\eta} O^{\prime}
$$

In addition, if $\tilde{\eta} \in O_{0}^{*}$ then $l_{0} \in I_{B^{\prime}}^{l o c}$.

Proposition 3.11. a) $[2](h) \equiv 2 \tilde{\eta}^{\prime} l_{0} \bmod 2\left(\tilde{\eta} I_{B^{\prime}}\right)^{l o c}$;

b) $\delta_{L T}(h) \equiv \tilde{\eta}^{\prime} \delta^{+}\left(l_{0}\right) \bmod \left(\tilde{\eta}^{\prime} I_{B^{\prime} \otimes B^{\prime}}(4)\right)^{l o c}$.

Proof. a) By Main Lemma, $h \in I_{B^{\prime}}(2)^{l o c}$ and, therefore, [2] $(h) \in$ $2 I_{B^{\prime}}(2)^{l o c}+\left(I_{B^{\prime}}(2)^{l o c}\right)^{2} \subset I_{B^{\prime}}(4)^{l o c}$, cf. Subsection 2.2.3. The standard DP-structure on this ideal is topologically nilpotent and $2\left(\tilde{\eta}^{\prime} I_{B^{\prime}}\right)^{l o c}$ is its DP-subideal. Therefore, the congruence $l_{L T}([2] h) \equiv 2 \tilde{\eta}^{\prime} l_{0} \bmod 2\left(\tilde{\eta} I_{B^{\prime}}(4)\right)^{l o c}$ implies that $[2](h) \in 2\left(\tilde{\eta}^{\prime} I_{B^{\prime}}\right)^{l o c}$ (use that $l_{L T}$ is bijective on any ideal with nilpotent divided powers). If $[2](h)=2 A$ with $A \in\left(\tilde{\eta}^{\prime} I_{B^{\prime}}\right)^{l o c}$ then $l_{L T}([2](h)) \equiv 2 A \bmod 2\left(\tilde{\eta} I_{B^{\prime}}\right)^{l o c}$ and, therefore, $A \equiv \tilde{\eta}^{\prime} l_{0} \bmod \left(\tilde{\eta} I_{B^{\prime}}\right)^{l o c}$.

b) Similarly, Main Lemma implies that

$$
\delta_{L T}(h) \in I_{B^{\prime}}(2)^{l o c} \otimes I_{B^{\prime}}(2)^{l o c} \subset I_{B^{\prime} \otimes B^{\prime}}(4)^{l o c} .
$$

Again this ideal has topologically nilpotent DP-structure and in addition we have

$$
l_{L T}\left(\delta_{L T}(h)\right)=\delta^{+} l_{L T}(h) \equiv \tilde{\eta}^{\prime} \delta^{+} l_{0} \bmod \left(\tilde{\eta} I_{B^{\prime} \otimes B^{\prime}}(4)\right)^{l o c}
$$

Proceeding as in a) we obtain $\delta_{L T}(h) \in\left(\tilde{\eta}^{\prime} I_{B^{\prime} \otimes B^{\prime}}(4)\right)^{l o c}$. It remains to note that then $\delta_{L T}(h)^{2} / 2 \in\left(\tilde{\eta} I_{B^{\prime} \otimes B^{\prime}}(4)\right)^{l o c}$ and, therefore, $l_{L T}\left(\delta_{L T}(h)\right) \equiv$ $\delta_{L T}(h) \bmod \left(\tilde{\eta} I_{B^{\prime} \otimes B^{\prime}}(4)\right)^{l o c}$.

Proposition 3.12. For $1 \leqslant i \leqslant u,\left(\sum_{l} o_{i l}^{\prime 2}\right) \eta_{i} \in \tilde{\eta}^{2} O_{0} \bmod 2 \tilde{\eta} O$.

Proof. We know that $[2](f) \in \tilde{\eta}^{2} I_{B_{0}}$. On the other hand,

$$
[2](f)=[2]\left(f_{0}\right)+[2](h)+\sum_{i, l}[2]\left(o_{i l}^{\prime} X_{i}\right),
$$

where $f_{0} \in \tilde{\eta} I_{B}$. Note that:

$[2]\left(f_{0}\right) \equiv f_{0}^{2} \bmod 2 \tilde{\eta} I_{B}$ implies that $[2]\left(f_{0}\right) \in \tilde{\eta}^{2} I_{B_{0}} \bmod 2\left(\tilde{\eta} I_{B}\right)^{l o c}$;

$[2]\left(o_{i l}^{\prime} X_{i}\right) \equiv\left[o_{i l}^{\prime 2} X_{i}^{2}\right]+\left[2 o_{i l}^{\prime} X_{i}\right] \bmod 2\left(\tilde{\eta} I_{B}\right)^{l o c}$ (use that all $\left.o_{i l}^{\prime 2} \in \tilde{\eta} O\right)$;

$[2](h) \equiv-\sum_{i, l}\left[2 o_{i l}^{\prime} X_{i}\right] \bmod 2\left(\tilde{\eta} I_{B^{\prime}}\right)^{l o c}$ by the above proposition. 
So,

$$
\sum_{i, l}\left[o_{i l}^{\prime 2} X_{i}^{2}\right] \in \tilde{\eta}^{2} I_{B_{0}} \bmod 2\left(\tilde{\eta} I_{B}\right)^{l o c} .
$$

Note that for all $i$ (use just that $\pi^{2}=\pi_{0}$ ),

$$
\begin{aligned}
& o_{i 0}^{\prime 2}, o_{i 2}^{\prime 2} \in \tilde{\eta} O_{0}+2 \tilde{\eta} O ; \\
& o_{i 1}^{\prime 2}, o_{i 3}^{\prime 2} \in \pi \tilde{\eta} O_{0}+2 \tilde{\eta} O ; \\
& X_{i}^{2} \in \eta_{i} I_{B_{0}}+2 I_{B} .
\end{aligned}
$$

This implies that:

$$
\begin{aligned}
& \sum_{i}\left(\left[o_{i 0}^{\prime 2} X_{i}^{2}\right]+\left[o_{i 2}^{\prime 2} X_{i}^{2}\right]\right) \equiv \sum_{i}\left(o_{i 0}^{\prime 2}+o_{i 2}^{\prime 2}\right) X_{i}^{2} \bmod \left(\tilde{\eta}^{2} I_{B_{0}}+2 \tilde{\eta} I_{B}\right) \\
& \sum_{i}\left(\left[o_{i 1}^{\prime 2} X_{i}^{2}\right]+\left[o_{i 3}^{\prime 2} X_{i}^{2}\right]\right) \equiv \sum_{i}\left(o_{i 1}^{\prime 2}+o_{i 3}^{\prime 2}\right) X_{i}^{2} \bmod \left(\tilde{\eta}^{2} I_{B_{0}}+2 \tilde{\eta} I_{B}\right)
\end{aligned}
$$

Clearly, $\sum_{i}\left(o_{i 0}^{\prime 2}+o_{i 2}^{\prime 2}\right) X_{i}^{2} \in \tilde{\eta} I_{B_{0}}+2 \tilde{\eta} I_{B}$ and $\sum_{i}\left(o_{i 0}^{\prime 2}+o_{i 2}^{\prime 2}\right) X_{i}^{2} \in$ $\pi \tilde{\eta} I_{B_{0}}+2 \tilde{\eta} I_{B}$. Therefore, (3.6) implies that for any $1 \leqslant i \leqslant u$, $\left(o_{i 0}^{\prime 2}+o_{i 2}^{\prime 2}\right) \eta_{i} \in \tilde{\eta} O_{0}+2 \tilde{\eta} O$ and $\left(o_{i 1}^{\prime 2}+o_{i 3}^{\prime 2}\right) \eta_{i} \in 2 \tilde{\eta} O$. This proves the proposition.

3.11. Introducing $\mathcal{M}=\mathcal{M}(G) \in \mathrm{MF}_{S}^{e}$. Choose a rings identification $\kappa_{S^{\prime} O^{\prime}}: S^{\prime} \bmod t^{e} \longrightarrow O^{\prime} \bmod 2$ such that $\left.\kappa_{S^{\prime} O^{\prime}}\right|_{S \bmod t^{e}}=\kappa_{S O}$. Consider $l_{0}=\sum_{i} a_{i} X_{i} \in I_{B^{\prime}}$ from Subsection 3.10 and set

$$
l_{1}=\sum_{i} b_{i} X_{i} \equiv\left(-l_{0}-\tilde{\eta}^{\prime} l_{0}^{2}-\cdots-\tilde{\eta}^{2^{n}-1} l_{0}^{2^{n}}-\ldots\right) \bmod 2\left(\tilde{\eta}^{\prime} I_{B^{\prime}}\right)^{l o c}
$$

Let $\tilde{s}^{\prime}, \alpha_{1}, \ldots, \alpha_{u} \in S^{\prime}$ be such that $\kappa_{S^{\prime} O^{\prime}}\left(\tilde{s}^{\prime} \bmod t^{e}\right)=\tilde{\eta}^{\prime} \bmod 2$ and $\kappa_{S^{\prime} O^{\prime}}\left(\alpha_{i} \bmod t^{e}\right)=b_{i} \bmod 2$ for $1 \leqslant i \leqslant u$.

Introduce $\mathcal{M}^{\prime}=\mathcal{M}^{\prime}(G)=\left(M^{\prime 0}, M^{\prime 1}, \varphi_{1}^{\prime}\right) \supset \mathcal{N}^{\prime}:=\mathcal{N} \otimes_{S} S^{\prime}$ such that $M^{\prime 0}=m^{\prime} S^{\prime} \oplus\left(N^{0} \otimes_{S} S^{\prime}\right), M^{\prime 1}=m^{\prime 1} S^{\prime}+\left(N^{1} \otimes_{S} S^{\prime}\right) \subset M^{\prime 0}$ with $m^{\prime 1}=\tilde{s}^{\prime} m^{\prime}+\sum_{i} \alpha_{i} n_{i}$, and $\varphi_{1}^{\prime}\left(m^{\prime 1}\right)=m^{\prime}$.

Proposition 3.13. a) $\mathcal{M}^{\prime} \in \mathrm{MF}_{S^{\prime}}^{2 e}$;

b) there is $\mathcal{M}=\mathcal{M}(G) \in \mathrm{MF}_{S}^{e}$ such that $\mathcal{M}^{\prime}=\mathcal{M} \otimes_{S} S^{\prime}$.

Proof. a) $\mathcal{M}^{\prime} \in \mathrm{MF}_{S^{\prime}}^{2 e}$ means that $t^{e} m^{\prime} \in M^{\prime 1}$, i.e. for $1 \leqslant i \leqslant u$, one has $t^{e} \tilde{s}^{-1} \alpha_{i} \equiv 0 \bmod \tilde{s}_{i}$ or, equivalently,

$$
\left(t^{e} \tilde{s}_{i}^{-1}\right) \alpha_{i} \equiv 0 \bmod \tilde{s} .
$$

Using (3.5) and the identification $\kappa_{S^{\prime} O^{\prime}}$ we can rewrite these congruences in the form $\left(\sum_{l} o_{i l}^{\prime}\right) \eta_{i}^{\prime} \equiv 0 \bmod \tilde{\eta}$ or equivalently, $\left(\sum_{l} o_{i l}^{\prime 2}\right) \eta_{i} \equiv$ $0 \bmod \tilde{\eta}^{2}$. Clearly, these conditions follow from Proposition 3.12 .

b) The criterion of the existence of a descent $\mathcal{M}$ of $\mathcal{M}^{\prime}$ to $S$ from Proposition 1.4 can be specified in our case in the following form: for all $1 \leqslant i \leqslant u, \alpha_{i} \in S \bmod \tilde{s}_{i} S^{\prime}$. 
Using the identification $\kappa_{S^{\prime} O^{\prime}}$ we rewrite these conditions in the form $\tilde{b}_{i} \bmod \tilde{\eta}_{i}^{\prime} \in O \bmod \tilde{\eta}^{\prime}$. By (3.5) we can replace them by $\sum_{l} o_{i l}^{\prime} \in$ $O \bmod \tilde{\eta}_{i}^{\prime} \tilde{\eta}^{\prime}$, then by $\sum_{l} o_{i l}^{\prime 2} \in O_{0} \bmod \tilde{\eta}_{i} \tilde{\eta}$ and finally by $\left(\sum_{l} o_{i l}^{\prime 2}\right) \eta_{i} \in$ $O_{0} \bmod 2 \tilde{\eta} O$. But this is given again by Proposition 3.12 .

3.12. Construction of isomorphism $\mathcal{G}_{O}(\mathcal{M}) \simeq G$. We know that $G=\operatorname{Spec} A$, where $A=B[X],[2](\tilde{\eta} X)=[2](f) \in\left(\tilde{\eta}^{2} I_{B_{0}}^{l o c} \subset\left(\tilde{\eta}^{2} I_{B}\right)^{l o c}\right.$ and $\delta_{L T}(\tilde{\eta} X)=\delta_{L T}(f) \in\left(\tilde{\eta} I_{B_{0} \otimes B_{0}}\right)^{l o c} \subset\left(\tilde{\eta} I_{B \otimes B}\right)^{l o c}$.

Let $A^{\prime}=A \otimes_{O} O^{\prime}$ and let $Z \in I_{A^{\prime}}$ be such that $\tilde{\eta}^{\prime} Z=[\tilde{\eta} X]-$ $\sum_{i, l}\left[o_{i l}^{\prime} X_{i}\right]$. Note that

$$
Z+\tilde{\eta}^{\prime-1} \sum_{i, l} o_{i l}^{\prime} X_{i} \equiv Z-l_{0} \equiv Z+l_{1} \equiv 0 \bmod \tilde{\eta}^{\prime} I_{A^{\prime}}
$$

Proposition 3.14. a) $\tilde{\eta}^{\prime} Z \in\left(\tilde{\eta}^{\prime} I_{A^{\prime}}(2)\right)^{l o c}$;

b) $\delta^{+}\left(Z-l_{0}\right) \equiv \tilde{\eta}^{\prime} I_{A^{\prime} \otimes A^{\prime}}(4)$;

c) $\tilde{\eta}^{\prime}\left(Z^{2} / 2\right) \equiv Z+l_{1} \bmod \left(\tilde{\eta}^{\prime} I_{A^{\prime}}(4)\right)^{l o c}$.

Proof. a) By Proposition 3.11a $),[2]\left(\tilde{\eta}^{\prime} Z\right)=[2](h) \equiv 2 \tilde{\eta}^{\prime} l_{0} \bmod 2\left(\tilde{\eta} I_{B^{\prime}}\right)^{l o c}$. This implies $\left(\tilde{\eta}^{\prime} Z\right)^{2} \in 2\left(\tilde{\eta}^{\prime} I_{A^{\prime}}\right)^{l o c}$, i.e. $\tilde{\eta}^{\prime 1 / 2} Z \in I_{A^{\prime}}(2)^{l o c}$. If $\tilde{\eta} \in O_{0}^{*}$ then a) is proved. Continue with the following congruence

$$
l_{L T}\left(\tilde{\eta}^{\prime} Z\right)=l_{L T}(h) \equiv \tilde{\eta}^{\prime} l_{0} \bmod \left(\tilde{\eta} I_{B^{\prime}}(4)\right)^{l o c}
$$

(cf. Proposition 3.10). It implies that

$$
Z-l_{0}+\tilde{\eta}^{\prime}\left(Z^{2} / 2\right)\left(1+\tilde{\eta} Z^{2} / 2\right) \in\left(\tilde{\eta}^{\prime} I_{A^{\prime}}(4)\right)^{l o c} .
$$

By (3.7) $\left(Z-l_{0}\right) / \tilde{\eta}^{\prime} \in I_{A^{\prime}}$ and $1+\tilde{\eta}\left(Z^{2} / 2\right) \equiv 1 \bmod \tilde{\eta}^{\prime} I_{A^{\prime}}$ is invertible (use that $\tilde{\eta} \notin O_{0}^{*}$ ). Therefore, $Z^{2} / 2 \in I_{A^{\prime}}$ and a) is completely proved.

b) By proposition $3.11 \mathrm{~b}), \delta_{L T}\left(\tilde{\eta}^{\prime} Z\right) \equiv \tilde{\eta}^{\prime} \delta^{+} l_{0} \bmod \tilde{\eta} I_{B^{\prime} \otimes B^{\prime}}(4)$. It remains to note that $Z \in I_{A^{\prime}}(2)$ implies that

$$
\delta_{L T}\left(\tilde{\eta}^{\prime} Z\right) \equiv \delta^{+}\left(\tilde{\eta}^{\prime} Z\right) \bmod \tilde{\eta} I_{A^{\prime} \otimes A^{\prime}}(4) .
$$

c) Iterating (3.8) we obtain

$$
\begin{aligned}
& \tilde{\eta}^{\prime}\left(Z^{2} / 2\right) \equiv Z-l_{0}-\tilde{\eta}^{\prime}\left(\tilde{\eta}^{\prime} Z^{2} / 2\right)^{2} \equiv Z-l_{0}-\tilde{\eta}^{\prime} l_{0}^{2}-\tilde{\eta}^{\prime 3}\left(\tilde{\eta}^{\prime} Z^{2} / 2\right)^{4} \\
& \equiv Z-l_{0}-\tilde{\eta}^{\prime} l_{0}^{2}-\cdots-\tilde{\eta}^{\prime 2^{n}-1} l_{0}^{2^{n}}-\cdots \equiv Z+l_{1} \bmod \left(\tilde{\eta}^{\prime} I_{A^{\prime}}(4)\right)^{l o c} .
\end{aligned}
$$

The proposition is proved.

Consider the ideals $J_{A^{\prime}}$ and $J_{A^{\prime} \otimes A^{\prime}}$, cf. Subsection 1.3.

Corollary 3.15. There is $\widetilde{Z} \in I_{A^{\prime}}$ such that

a) $\widetilde{Z} \equiv\left(\tilde{\eta}^{\prime} I_{A^{\prime}}(4)\right)^{l o c}$;

b) $\tilde{\eta}^{\prime}\left(\widetilde{Z}^{2} / 2\right) \equiv \widetilde{Z}+l_{1} \bmod \tilde{\eta}^{\prime} J_{A^{\prime}}$;

c) $\delta^{+}(\widetilde{Z}), \delta^{+}\left(\widetilde{Z}^{2} / 2\right) \in J_{A^{\prime} \otimes A^{\prime}}$. 
Proof. Let $\widetilde{Z}=\tilde{\eta}^{\prime}\left(Z^{2} / 2\right)-l_{1}$.

Part a) follows directly from Proposition 3.14.

Because $\left(\tilde{\eta}^{\prime} I_{A^{\prime}}(4)\right)^{l o c} \subset I_{A^{\prime}}(2)$ this implies that

$$
\widetilde{Z}^{2} / 2 \equiv Z^{2} / 2 \bmod J_{A^{\prime}}
$$

and we obtained part b).

By Proposition $3.14 \mathrm{~b}), \delta^{+} Z \in I_{A^{\prime} \otimes A^{\prime}}(4)$. This implies that $\delta^{+}\left(Z^{2} / 2\right) \in$ $J_{A^{\prime} \otimes A^{\prime}}$, use Proposition 3.14a). So, $\delta^{+} Z \in J_{A^{\prime} \otimes A^{\prime}}$ and by (3.9) we obtain $\delta^{+}\left(Z^{2} / 2\right) \in J_{A^{\prime} \otimes A^{\prime}}$.

By above Corollary the correspondences $m^{\prime} \mapsto \widetilde{Z}^{2} / 2 \bmod J_{A^{\prime}}$ and $m^{\prime 1} \mapsto \widetilde{Z} \bmod J_{A^{\prime}}$ give a map of filtered modules $\mathcal{M}^{\prime} \longrightarrow \iota\left(A^{\prime}\right)$. This gives a morphism of $O^{\prime}$-algebras $\Pi^{\prime}: A\left(\mathcal{G}_{O^{\prime}}\left(\mathcal{M}^{\prime}\right)\right) \longrightarrow A^{\prime}$. By Proposition 1.12 we can assume that $\Pi^{\prime}$ is also a morphism of coalgebras. Both these coalgebras contain $B^{\prime}=B \otimes_{O} O^{\prime}$ and $\left.\Pi^{\prime}\right|_{B^{\prime}}$ is isomorphism. Similarly, both the coalgebras have as their quotient the coalgebra $A\left(\mu_{\eta}\right) \otimes O^{\prime}$ and $\Pi^{\prime}$ induces on it a coalgebra isomorphism as well. This implies that $\Pi^{\prime}$ is isomorphism of coalgebras and Theorem 3.1 is completely proved.

\section{REFERENCES}

[1] V. AbRashkin Group schemes of period p, Math. USSR Izvestiya 20 (1983) no. 3, 411-433

[2] V. AbRAShKin Honda systems of group schemes of period p, Math. USSR Izvestiya 30 (1988) no. 3, 419-453

[3] V. ABRASHKIN Group schemes over a discrete valuation ring with small ramification, Leningrad Math. J. 1 (1990) no. 1, 1990, 57-97

[4] V. Abrashkin Group schemes of period $p>2$, Proc. London Math. Soc. 101 (2010) no. 3, 207-259

[5] C. Breuil, Groupes p-divisibles, groupes finis et modules filtrés, Ann. of Math. 1522000 no. 2, 489-549

[6] W. KIM The classification of p-divisible groups over 2-adic discrete valuation rings, arXiv:1007.1904. Preprint.

[7] M. KisIn Modularity of 2-adic Barsotti-Tate representations, Inv. Math. 178 (2009), 587-634

[8] M. KIsIN Moduli of finite flat group schemes, and modularity, Ann. of Math. 170 (2009), 1085-1180

[9] E. LAU, A relation between crystalline Dieudonné theory and Dieudonné displays, arXiv:1006.2720. Preprint.

[10] T. Liu, The correspondence between Barsotti-Tate groups and Kisin modules when $p=2$, available at math.purdue.edu/tongliu/pub/2BT.pdf. Preprint.

Department of Mathematical Sciences, Durham University, Science Laboratories, South Rd, Durham DH1 3LE, United Kingdom \& Steklov Mathematical Institute, Gubkina str. 8, 119991, Moscow, Russia

E-mail address: victor.abrashkin@durham.ac.uk 